\title{
DE L'HÔPITAL À L'ÉTAT : LE REGARD ETHNOGRAPHIQUE AU CHEVET DE L'ACTION PUBLIQUE
}

\section{Nicolas Belorgey}

\section{Presses de Sciences Po (P.F.N.S.P.) | «Gouvernement et action publique »}

$2012 / 2 \mathrm{n}^{\circ} 2 \mid$ pages 9 à 40

ISSN 2260-0965

ISBN 9782724632910

Article disponible en ligne à l'adresse :

http://www.cairn.info/revue-gouvernement-et-action-publique-2012-2-page-9.htm

\section{Pour citer cet article :}

Nicolas Belorgey, « De l'hôpital à l'état : le regard ethnographique au chevet de l'action publique », Gouvernement et action publique 2012/2 ( $\left.{ }^{\circ} 2\right)$, p. 9-40. DOI 10.3917/gap.122.0009

Distribution électronique Cairn.info pour Presses de Sciences Po (P.F.N.S.P.).

(C) Presses de Sciences Po (P.F.N.S.P.). Tous droits réservés pour tous pays.

La reproduction ou représentation de cet article, notamment par photocopie, n'est autorisée que dans les limites des conditions générales d'utilisation du site ou, le cas échéant, des conditions générales de la licence souscrite par votre établissement. Toute autre reproduction ou représentation, en tout ou partie, sous quelque forme et de quelque manière que ce soit, est interdite sauf accord préalable et écrit de l'éditeur, en dehors des cas prévus par la législation en vigueur en France. Il est précisé que son stockage dans une base de données est également interdit. 


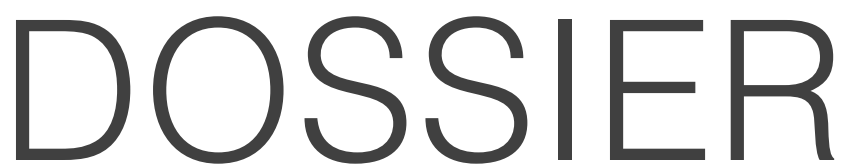

Approches croisées de l'État, du gouvernement et de l'action publique

De l'hôpital à l'État : le regard ethnographique au chevet de l'action publique

État, administration et politiques publiques : les dé-liaisons dangereuses

Qui gouverne les politiques publiques? 


\title{
DE L'HÔPITAL À L'ÉTAT : LE REGARD ETHNOGRAPHIQUE AU CHEVET DE L'ACTION PUBLIQUE
}

Nicolas Belorgey

Résumé : Ce texte propose de croiser systématiquement méthode ethnographique et analyse de l'action publique. Quatre niveaux d'étude d'une action publique sont à ce titre définis: ceux des destinataires de cette action, de ses acteurs publics des premiers et seconds rangs (streel-level bureaucrats et intermédiaires multiples), enfin de ses concepteurs. II montre dans une première partie que l'analyse de l'action publique a évolué peu à peu vers plus de réalisme, par une réduction (limitée) de son ethnocentrisme de position initial, par une critique (incomplète) du modèle de l'homo oeconomicus, enfin par un (relatif) déplacement méthodologique. II montre ensuite que, bien qu'elle lui soit encore peu appliquée, l'ethnographie est un mode de connaissance propice à l'étude de l'action publique : elle permet de dépasser l'ethnocentrisme de position, de mobiliser tous les outils des sciences sociales, de ne pas ignorer certaines dimensions essentielles des acteurs, comme la trajectoire sociale, la génération ou le genre. Enfin ce texte illustre ce croisement en donnant quelques méthodes, «ficelles » et résultats obtenus à propos de l'action publique hospitalière dans les années 2000.

MOTS-CLÉS : ACTION PUBLIQUE - ÉTAT - ETHNOGRAPHIE - HÔPITAL - MÉTHODOLOGIE - NOUVELLE GESTION PUBLIQUE

\begin{abstract}
This article seeks to enrich the analysis of public action, or policy, by blending it with an ethnographic method. To this end, it defines four levels of public action: 1) the targets of policy; 2) "street-level" practitioners and 3) their immediate supervisors; and finally 4) those at the origin of the policies in question. In a first section, it demonstrates that the analysis of public policy has gradually evolved toward a position of greater realism through a (limited) reduction of its initial ethnocentrism, by an (incomplete) critique of the model of "economic man", and finally through a (relative) methodological shift. It further demonstrates that, although it is as yet little applied, ethnography is a type of knowledge that is useful for the study of public policy: it allows us to overcome the problem of positional ethnocentrism and to mobilize all of the tools of social science without ignoring certain essential dimensions of actors such as their age, gender, and social trajectory. Ultimately, this article illustrates this hybrid approach through examples of methods and results obtained in the study of French public policy in the hospital sector in the 2000s.
\end{abstract}

KEYWORDS: ETHNOGRAPHY - HOSPITAL - METHODS - NEW PUBLIC MANAGEMENT - PUBLIC ACTION - STATE - STREET-LEVEL BUREAUCRACY

De manière récurrente depuis une quinzaine d'années, émerge l'idée d'un certain « essoufflement » de l'analyse des politiques publiques en France (Bezes, Pierru, 2012, introduction). 
On voudrait ici suggérer non une recette miracle, mais quelques moyens de ré-oxygénation, sous la forme de techniques d'enquête éprouvées dans d'autres domaines, qui n'ont pas encore été appliquées pleinement aux politiques publiques, ou ne le sont plus suffisamment : celles de l'ethnographie. L'analyse de l'action publique se définissant par son objet, aucune méthode ne lui est a priori interdite ${ }^{1}$. Réciproquement, l'ethnographie étant un mode de connaissance du monde social en général, rien n'empêche en principe de l'appliquer à cet objet particulier. Pourquoi, dès lors, ces deux disciplines, l'une attachée à élucider pleinement une partie du monde social, l'autre à comprendre celui-ci en général d'une certaine façon, ne se sont-elles pas davantage rencontrées ? Vraisemblablement à cause des biais propres à chacune d'entre elles. L'analyse de l'action publique a certes beaucoup évolué, comme le signale cette nouvelle appellation, qui tend à remplacer celle de " politiques publiques ", désormais trop stato-centrée. Elle trouve cependant son origine dans l'analyse de "décisions ", de surcroît initialement supposées rationnelles, à l'aide de disciplines relativement liées à l'État, théoriques ou normatives, comme l'histoire événementielle, le droit public ou la théorie de l'action rationnelle. Dans ces conditions, une approche ne serait-ce que sociologique y a longtemps fait figure de curiosité exotique. En France, une méthode telle que l'entretien est demeurée restreinte dans ses modalités d'utilisation, tandis que l'observation, a fortiori participante, en était quasiment absente (Bongrand, Laborier, 2005). Comme le notent des travaux récents (Biland, 2008, p. 23), «l'analyse des politiques publiques demeure souvent insuffisamment attentive aux acteurs et à leurs trajectoires". La discipline semble peiner encore aujourd'hui à mobiliser les acquis du raisonnement sociologique, notamment l'existence de relations de domination au sein de la société (Dubois, 2009).

Réciproquement, l'ethnographie est initialement très distante de ce qui ne se nomme alors même pas encore les politiques publiques. Les travaux qui en relèvent s'intéressent d'abord aux sociétés lointaines, conçues comme radicalement autres; ils sont attachés à les décrire et considèrent volontiers l'État comme une spécificité occidentale, au mieux comme faiblement autonomisé, immergé dans des faits sociaux plus généraux qui sont, eux, l'objet pertinent pour comprendre la réalité sociale (notammant Mauss, 1923 ; Evans-Pritchard, 1937 ; Sahlins, 1972). Certes, des approches inspirées de l'ethnographie sont appliquées par des auteurs occidentaux dans leur propre société de manière réflexive dès les années 1930 puis après la seconde guerre mondiale, par exemple par les sociologues de Chicago. Mais les deux disciplines demeurent séparées. Par exemple, les célèbres études d'Howard Becker (1963) ou de Joseph Gusfield (1966) adoptent des entrées théoriques beaucoup plus sociologiques (la déviance, les croisades symboliques) que celles, centrées autour de la décision, qui prévalent alors dans l'étude des politiques publiques (notamment Jones, 1970). En outre, elles peinent à appliquer les outils de l'entretien et de l'observation directe au milieu fermé des décideurs. Enfin, la tradition de Chicago est quelque peu noyée aujourd'hui aux États-Unis au milieu de l'hyperspécialisation des sous-disciplines qui y prévaut en sociologie et en science politique, elles-mêmes nettement cloisonnées (Bezes, Pierru, 2012). Les chercheurs étasuniens qui mobilisent les outils de l'ethnographie dans l'analyse des politiques publiques sont aujourd'hui peu nombreux et ne les appliquent pas au milieu des décideurs (par exemple Stack, 1997). De manière analogue, une bonne partie des

1. "The policy orientation therefore may be summed up in Harold Lasswell's term as being: multi-method, multi-disciplinary, problem-focused » (Parsons 1995, p. xvi). 
ethnographes et des sociologues français négligent l'étude de l'action publique, volontiers assimilée à son courant cognitif (comme le remarquent Bongrand, Laborier, 2005, p. 82), dont ils sont très distants dans le champ des sciences sociales. Pour reprendre la formule de l'un d'entre eux, l'ethnographie ne s'intéresse pas initialement aux sommets de l'État, mais entend au contraire " aller voir comment vivent ceux qui ne parlent pas assez fort pour couvrir le bruit que font les puissants " (Weber, 2009, p. 1).

Dans ces conditions, étudier ethnographiquement l'action publique procède d'un double pari : que le renouveau de cette discipline est à trouver notamment dans un élargissement méthodologique déjà esquissé mais jamais complètement réalisé ; que l'ethnographie a également tout intérêt à renouveler ses objets d'étude classiques, ou pour prolonger la citation précédente (p. 2), à désormais aussi « aller voir ce que font sans bruit les puissants ».

Mener à bien une telle démarche suppose de préciser ce dont on parle. L'ethnographie peut se définir comme une enquête directe par interconnaissance dans certains milieux, mobilisant les outils de l'observation et de l'entretien (Maget, 1953 ; Beaud, Weber, 1997 ; Weber, 2009). L'étude de l'action publique de son côté, initialement étude de «l'État en action ", est devenue aujourd'hui avec la multiplication des acteurs concernés et le relatif recul de cet État, dans le temps long de l'histoire, par rapport aux acteurs locaux, supranationaux et privés, une " analyse contextualisée d'interaction d'acteurs multiples et enchevêtrés à plusieurs niveaux, du local à l'international [...], permettant de penser les transformations des États contemporains " (Hassenteufel, 2008, p. 23). Croiser ces deux définitions implique de caractériser les différents types d'acteurs en présence en les situant non plus par rapport à l'État, mais par rapport à l'action publique elle-même qu'on entend élucider. Toute action publique comporte à ce titre quatre niveaux dont aucun ne doit être négligé pour aboutir à une compréhension d'ensemble, et qui sont ici illustrés sur un cas d'espèce, celui de l'action publique menée dans les hôpitaux. Le premier de ces quatre niveaux est celui de la conception de cette politique ou du moins, si aucune cohérence ou intention n'y préside, celui des instances qui édictent les règles qui ont le plus de chances de s'imposer effectivement aux acteurs concernés. Par exemple, dans le domaine hospitalier: les " tutelles " (État, CNAM, agences et autorités diverses) et les normes juridiques, morales, techniques, comptables, organisationnelles, budgétaires, etc., qui prévalent dans les établissements. Ce niveau de l'action publique est loin d'être homogène et irénique. Les conflits y sont à la hauteur des enjeux que constitue la définition de normes qui ensuite auront pour elles la force du droit positif. Ces règles sont souvent des compromis temporaires entre factions durablement rivales. Elles sont aussi parfois elles-mêmes pétries de contradictions. Ainsi, si une « politique hospitalière » pérenne, globale et cohérente semble un objet problématique, on peut identifier au moins certains programmes d'action, comme les plans "Hôpital 2007 » ou " Hôpital 2012 " promus successivement par le ministère, ainsi que des changements normatifs majeurs (de niveau législatif). Nous désignons ce premier niveau d'action publique comme celui de la définition des normes légitimes du secteur.

À l'autre bout de la chaîne se trouvent, dans le cas des hôpitaux, les patients. Everett Hughes (1956, p. 305) demandait déjà malicieusement si l'on devait dire que dans ces établissements des choses étaient faites à eux ou pour eux. Le langage courant et le vocabulaire classique de la science politique font preuve de la même hésitation pour désigner ce niveau 
d'analyse de l'action publique en général. Assujettis, administrés, "ressortissants " (Warin, 1999) d'une politique publique, cible, "public visé » (Hassenteufel, 2008, p. 10), ou bien à l'inverse bénéficiaires d'une politique, ayants droit, " usagers de services publics » (Avril et al., 2005), "habitants " d'un quartier (Siblot, 2006), citoyens... : dans un cas la légitimité vient d'une autorité et la politique menée n'est pas nécessairement agréable aux personnes, dans l'autre ce sont les personnes qui légitiment cette politique, dont le résultat doit être jugé à leur aune ${ }^{2}$. La lancinante question de Hughes peut ainsi être étendue à tous les "individus auxquels s'adressent les autorités étatiques quand elles agissent ", comme les définit Patrick Hassenteufel (2008, p. 10), qui fournit peut-être à cette occasion la formulation la plus neutre. Nous inscrivant comme cette formule dans un champ sémantique communicationnel, nous les nommons par raccourci les destinataires de l'action publique.

Entre eux et le niveau de l'édiction des normes, se trouvent d'autres types d'acteurs que l'on ne saurait ignorer. II s'agit d'abord des acteurs publics du premier rang (les streetlevel bureaucrats de Michael Lipsky, 1980), au contact direct des destinataires de la politique. Enseignants, policiers, juges de première instance, soignants (médecins, infirmières, aidessoignantes, etc.), agents des caisses d'allocations familiales, guichetiers de multiples administrations, etc., ils sont ceux dont les faits et gestes font en pratique la politique publique concernée, par exemple, dans le contexte historique étudié par l'auteur, ceux qui contribuent involontairement à dissuader les bénéficiaires potentiels du public welfare de recourir à celui-ci (une actualisation est fournie par Brodkin, 2011). Nous les nommons les acteurs du premier rang de la politique publique concernée.

Enfin, une action publique ne peut avoir lieu sans l'intervention de personnes qui jouent le rôle d'intermédiaires (Nay, Smith, 2002) entre ces acteurs du premier rang et le niveau de la conception, autrement dit dont l'action est nécessaire pour que les normes édictées à cet endroit ne restent pas lettre morte. Directeurs d'hôpitaux, chefs de service de toutes les administrations, cadres, contrôleurs chargés du respect des normes, membres d'inspections centrales, auditeurs, etc., ces acteurs du second rang ne sont généralement pas au contact direct du public mais doivent s'assurer que les personnes du premier rang font bien leur travail conformément aux instructions multiformes émanant du niveau des instances normatives. Ils transmettent aussi, en sens inverse, ce qui ressort du travail des acteurs du premier rang au niveau de la conception des normes. Leur intermédiation se fait dans les deux sens.

Destinataires, acteurs du premier rang, acteurs du second rang ou intermédiaires, auteurs de normes (à commencer par l'État), forment donc les quatre niveaux qui composent toute politique publique, et qui peuvent être lus à travers différents travaux que ce texte détaille. Certes, il n'y a pas entre eux de barrière parfaitement étanche. Les personnes peuvent exercer plusieurs rôles à la fois, comme un chef de service qui continue à être un soignant. Elles peuvent aussi passer d'un rôle à un autre, comme les acteurs du premier ou du second rang investis dans des fonctions collectives, syndicales par exemple, qui contribuent de manière temporaire ou plus durable à la définition des normes. Mais cela n'enlève

2. "Client » (par exemple, Lipsky, 1980), peut être interprété dans les deux sens mais se situe dans un registre privé qui ignore le caractère public de l'action. 
rien à l'existence de ces quatre niveaux, et les distinguer avec leurs spécificités puis les étudier de concert permet de décrire l'ensemble d'une action publique, de la saisir non pas comme une intention plus ou moins bien réalisée, mais par regroupement de tous les types d'actions qui la composent. Elle peut être alors lue comme une configuration la plus complète possible d'acteurs interdépendants (Elias, 1991, p. 10, 154-161) ${ }^{3}$.

Au croisement de préoccupations épistémologiques et méthodologiques, ce texte propose d'appliquer l'approche ethnographique à l'étude de l'action publique. II procède pour cela en trois temps. II montre d'abord que la seconde aurait tout à y gagner en termes de réalisme. II montre ensuite que la première est un outil propice à rendre compte des actions publiques, mais encore insuffisamment mobilisée sur cet objet relativement nouveau pour elle. II illustre enfin ces éléments par un cas d'espèce, auquel il a déjà été fait allusion dans cette introduction: l'action publique hospitalière en France au cours des années 2000 (Belorgey, 2010). II entend ainsi généraliser à l'étude de l'État et de l'action publique la démarche esquissée lors de celle des hôpitaux.

Cet objet est en effet propice à une telle démarche. Dans un contexte de retrait de l'État social par la réduction de ses financements (Duval, 2002, 2007), une certaine coalition d'intérêts s'efforce de promouvoir au niveau collectif un ensemble de normes nouvelles. Ce processus réformateur (Becker, 1963 ; Topalov, 1999) ${ }^{4}$ consiste notamment en la montée en puissance du raisonnement économique dans la sphère de la santé (Benamouzig, 2005) et en l'avènement d'une nouvelle séquence réformatrice, celle du New Public Management (Bezes, 2009). Le secteur de la santé, et notamment les hôpitaux, se trouvent ainsi pris entre les feux croisés de deux libéralismes (Pierru, 2007) : alors que le libéralisme de combat des médecins fait reculer les pouvoirs publics, notamment pour des raisons électoralistes, un néolibéralisme est à l'œuvre dans les hôpitaux, dans le sens où l'État organise lui-même son propre retrait, l'étatisation servant de levier pour la libéralisation et la privatisation. On est donc bien face à un cas exemplaire de transformation de l'État contemporain, dans le sens où celui-ci demeure particulièrement actif mais où cette action ne se comprend qu'en la replaçant dans une configuration d'acteurs plus large, tant publics que privés.

\section{L'analyse de l'action publique : une discipline qui évolue peu à peu vers un plus grand réalisme}

Parce que les politiques publiques elles-mêmes ont changé, et par l'importation de nouvelles méthodes depuis différents horizons des sciences sociales, l'analyse de l'action publique s'est considérablement renouvelée depuis sa naissance. Globalement, l'histoire de

3. Comme l'indiquent Stéphane Beaud et Florence Weber (1997, p. 302), le concept de chaîne d'interdépendance chez Elias est lui-même une généralisation de celui de schismogénèse chez l'ethnographe Bateson (1936). Ainsi, cette définition eliasienne de l'action publique est directement inspirée de la méthode ethnographique. Une application récente en a été faite par Sylvain Laurens (2009) à propos des hauts fonctionnaires qui font la politique de l'immigration, maillon intermédiaire mais décisif dans cette chaîne.

4. À la différence des auteurs de cette entreprise, qui se prévalent du sens commun du terme de "réforme " et de ses dérivés (" amélioration des choses dans le domaine moral ou social ", écrit le Petit Robert, 1999), dans ce texte, ceux-ci sont utilisés dans ce sens sociologique : une volonté de modifier certaines normes, portée par une coalition d'intérêts. 
la discipline peut être lue comme une déconstruction progressive du mythe du décideur rationnel, omniscient et omnipotent. Cependant, ce renouvellement n'a pas touché de manière égale toutes ses composantes. Certains de ses courants font toujours preuve d'un certain ethnocentrisme de position, ne fournissent qu'une critique incomplète du modèle épistémologique de l'homo oeconomicus, et n'utilisent qu'une partie des outils d'enquête qui s'offrent à eux.

\section{Une réduction limitée de l'ethnocentrisme de position}

On peut définir l'ethnocentrisme de position, dans l'analyse de l'action publique, comme la tendance à appréhender celle-ci à partir du seul niveau de sa conception ou de l'édiction des normes. De même que l'ethnocentrisme est le premier mouvement de toute perception de l'altérité culturelle (Lévi-Strauss, 1952), ou que l'ethnocentrisme de classe répète ce mouvement entre groupes sociaux inégalement dotés en ressources (Grignon, Passeron, 1989), l'ethnocentrisme de position est le premier mouvement de la perception d'une politique publique. Pensée archétypique de celle-ci par ses auteurs, il la considère sinon comme un tout cohérent, une réponse fonctionnelle à un problème dûment identifié au préalable, du moins comme se comprenant à la lumière des problèmes qui se posent aux gouvernants et dans le cadre de leurs catégories de pensée. Dans son optique, les trois autres niveaux de l'action publique sont perçus, quand ils n'assurent pas une bonne exécution des instructions, comme autant de dysfonctionnements, de foyers d'irrationalité ou de «boîtes noires ».

Si ce mouvement de pensée se rencontre encore couramment aujourd'hui, c'est surtout dans l'histoire de la discipline qu'il est présent. Celle-ci mêle en effet étroitement à son origine préoccupations descriptives et normatives. Elle est d'abord héritière des études de policy science américaines, où il s'agissait au moins autant de fournir des aides à la décision que de rendre compte d'une réalité 5 . C'est pourquoi elle s'est d'abord intéressée à l'analyse de "la décision ", selon des canons solidifiés par des auteurs tels que Harold Lasswell (1956) ou Charles Jones (1970). Celle-ci apparaît alors dans une séquence plus longue (préparation, décision, exécution, évaluation, etc.) comme le moment fort de l'analyse, laquelle s'efforce d'éclairer le décideur pour qu'il puisse prendre la meilleure décision possible. Son importation en France, par la suite, a conservé cette ambivalence constitutive (Dubois, 2009).

Particulièrement représentatif de ce temps de la réflexion est l'ouvrage qui, paradoxalement, est parfois cité aujourd'hui comme l'ayant au contraire ouverte vers d'autres horizons. II s'agit du livre de Jeffrey Pressman et Aaron Wildavsky (1973), dont le titre - « Mise en œuvre » (Implementation) - suggère au contraire que la focale serait enfin déplacée du niveau de la décision vers celui des acteurs des autres niveaux de la politique publique concernée.

5. Comme le remarque Patrick Hassenteufel (2008, p. 19), « les premiers analystes des politiques publiques sont en même temps des praticiens de l'action publique ». "Les policy sciences s'appuient [...] sur une représentation de l'action étatique tournée vers l'opérationnel » (ibid., p. 78). "L'approche de Lindblom [cf. infra] [...] même si elle se présente comme une approche "réaliste", pose le problème d'être également normative " (ibid.). Cette ambivalence est toujours présente dans les manuels étasuniens. L'un des ouvrages de référence en la matière (Parsons, 1995) porte explicitement comme sous-titre « An introduction to the theory and practice of policy analysis » et explique (p. xvi) que « in the book we mix two kind of approach which are often separated out in textbooks: the analysis of the policy process, and the use of analytical techniques and knowledge for and in policy-making ". 
Mais tout l'ouvrage reflète un ethnocentrisme de position d'autant plus prégnant qu'il prétend justement s'être affranchi du préjugé décisionniste. L'action publique analysée, lancée en 1965 dans le cadre du projet de Grande société du président démocrate L. Johnson, vise à donner du travail aux membres de la minorité noire de la ville d'Oakland, massivement pauvres et au chômage. Cette action éminemment louable incite d'emblée le lecteur à s'identifier aux décideurs justiciers et à faire siens leurs problèmes. Ceux-ci seraient principalement dus à la foule des acteurs en présence : multiples administrations fédérales situées à Washington, municipalité républicaine d'Oakland, nombreuses commissions ad hoc... Les auteurs dénoncent la multiplicité des instances et des autorisations (clearances) requises pour passer du principe de création d'emplois par une politique de grands travaux et de prêts aux entreprises à la réalisation de celle-ci. Les destinataires de la politique, membres de la minorité noire, ne sont évoqués qu'à quelques rares reprises, qui rappellent comme en passant que le problème de fond est la réticence des entreprises locales, détenues par l'establishment blanc, à les employer. Mais sur les mécanismes de cette ségrégation pourtant fondatrice du "problème ", ainsi que sur cette population, les seuls matériaux disponibles résident dans quelques statistiques et la mention de l'existence de quelques leaders noirs. Les enquêteurs, enrôlés par les promoteurs de l'action, ne sont jamais allés au contact de ses destinataires. Ainsi cet ouvrage reflète clairement un ethnocentrisme de position.

La même formulation à front renversé apparaît en France, par exemple chez Lucien Sfez. D'un côté, celui-ci critique fermement la notion de décision et le point de vue du décideur, comme l'indique le titre de son premier ouvrage, Critique de la décision (1973). De l'autre côté, la théorie alternative qu'il propose pour rendre compte de l'action publique, celle du surcode, demeure proche des catégories de perception des gouvernants. Selon cette théorie, inspirée de la linguistique et de la psychanalyse particulièrement en vogue dans les années 1970, le changement interviendrait certes, non grâce à une décision démiurgique, mais dans les lieux " où une information tronquée, traduite, déformée, passe d'un soussystème à un autre et peut être transmise et mise en pratique » (p. 470), ce qui peut sembler minorer le rôle du décideur. Mais cette "explication » est bien utilisable par les entrepreneurs de morale du niveau de la conception des normes. Elle semble répondre à la question : comment moi, en situation de décision, puis-je faire prévaloir ma volonté malgré les obstacles de la réalité extérieure ? La réponse est inspirée de la psychanalyse : " Faire usage du schéma de la pulsion inconsciente cherchant à faire sa voie à travers les censures [...]. Un projet est traversé, latéralisé par le système voisin. Cependant il se réalise... par la bande ${ }^{6}$. "

Si certains travaux semblent ainsi toujours répondre plus ou moins directement aux préoccupations des décideurs ou des intermédiaires qui les relaient, d'autres, à partir des années 1980 environ, appellent à dépasser ce modèle, mais d'une manière récurrente, qui jette un doute sur l'effectivité de ce dépassement, ou même sur sa possibilité si on se limite aux outils traditionnels de la discipline. II y a environ un quart de siècle (1986), Paul Sabatier publiait en effet un article très théorique dont on peut traduire le titre en français par : "Approches par le haut (top-down) et par le bas (bottom-up) de la mise en œuvre : analyse critique

6. À ce titre, L. Sfez est peut-être un chaînon intermédiaire entre la psychanalyse et les recettes de certains consultants privés actuels qui parlent également de « latéralisation " d'un projet pour le faire accepter par ses opposants (Belorgey, 2010, chap. 5). 
et tentative de synthèse ". Une recherche récente (Musselin, 2005), reprise par un manuel (Lascoumes, Le Galès, 2007, p. 14-15), semble lancer le même appel. Dans une volonté de synthèse de ce type d'approche, ce manuel la décrit dans le vocabulaire de la théorie économique de l'agence (notamment Fama, 1980) : elle aurait montré « à quel point le "principal" (la puissance publique) est confronté aux stratégies autonomes de ses "agents" » (p. 44). II s'agit donc bien, toujours dans le style du modèle du décideur, de chercher à mettre en œuvre les décisions du " principal » en ne concevant les « agents » que comme leurs relais potentiels et plus ou moins efficaces. Tout ce qui pourrait écarter du bon accomplissement des instructions qu'ils reçoivent est, comme dans le modèle économique sous-jacent où le résultat dépend du niveau d'" effort " des agents et de leur "performance " individuelle, imputé à des défauts de ceux-ci. Ainsi, si l'action publique aboutit à des résultats étranges, ce ne pourrait être en raison de ses concepteurs, mais seulement du fait de l'impéritie des acteurs responsables de son exécution. Même si elle se propose de procéder " par en bas ", cette approche demeure donc orientée par un regard jeté "par en haut ", et emporte donc à la fois une dimension normative et une portée épistémique limitées, du fait même de cette normativité. La répétition de l'appel à dépasser le vieux modèle décisionnel, ou top down, qu'on enrichit peu à peu mais dont on conserve l'approche, ne débouche pas, à l'heure actuelle, sur une élucidation de ce qui se passe au niveau des destinataires de l'action publique, ni même à celui des acteurs du premier ou du second rang.

\section{Une critique incomplète du modèle épistémologique de l'homo oeconomicus}

Centrés sur les entrepreneurs de normes, les travaux d'analyse de l'action publique existants font parfois aussi encore preuve, comme l'indique la référence au modèle principal-agent, d'une reprise persistante de certaines hypothèses de l'homo oeconomicus: individualisme méthodologique, exogénéité des préférences individuelles ${ }^{7}$. Non que certains d'entre eux n'aient développé une critique à leur égard, mais celle-ci n'est jamais allée jusqu'à remettre en cause le paradigme économique, lui demeurant interne. Ainsi, la sociologie des organisations a, parmi d'autres, largement repris l'assouplissement de l'hypothèse économique de rationalité substantive en rationalité limitée des acteurs (au sens de March, Simon, 1958). Autre exemple : le courant cognitif de la discipline (Muller, 1990), en intégrant dans son analyse les idées et les représentations des acteurs aux côtés des déterminants matériels de leurs actes, a suivi en cela le même chemin épistémologique que la théorie économique standard, passée d'une approche purement matérialiste à l'approche formelle plus large de la maximisation sous contrainte (sur cette évolution, cf. notamment M. Weber, 1922 ; Kolm, 1986 ; Demeulenaere, 1996). Ainsi, les œuvres parfois présentées comme les plus originales ou les plus critiques dans la discipline demeurent malgré tout informées par ce paradigme, comme l'illustre particulièrement le modèle de la poubelle.

De même que celui du muddling through (Lindblom, 1959), celui-ci (Cohen et al., 1972) demeure en effet désocialisé. II se présente certes comme une volonté de rupture, en cette année 1972, avec le classique modèle d'optimisation de la décision. Cela se voit

7. Le terme économique d'exogénéité signifie que les éléments en question sont reconnus dans leur existence mais non expliqués. Par exemple : les modèles de croissance à progrès technique exogène. 
notamment à son titre provocateur ou à l'oxymore par lequel il commence ("Consider organized anarchies"). Mais il partage malgré tout avec ce modèle d'origine économique un certain nombre de traits. Tout d'abord, deux des principaux paramètres qu'il identifie comme à même de faire varier ses résultats concernent le niveau de ressources, absolu ou relatif, des organisations. Celles-ci sont en effet décrites comme pauvres ou riches, et dotées d'un slack, c'est-à-dire d'un écart entre ce niveau de ressources et les besoins qu'elles doivent satisfaire, plus ou moins grand. Ensuite, l'hypothèse selon laquelle ce sont les problèmes demandant encore le moins de travail qui sont traités en priorité (energy allocation assumption, p. 4), suppose implicitement que les acteurs accordent a priori la même importance à tous les problèmes, ce qui n'est guère réaliste ${ }^{8}$. Enfin, l'article ne donne pas la source de ses données empiriques; il indique seulement à ce sujet que les propriétés dont il parle " ont été observées souvent dans les études sur les organisations " (p. 1). II peut donc être considéré comme une hypothèse posée sur le fonctionnement, vraisemblablement, des universités américaines dans les années 1960 ou au début des années 1970. II est totalement abstrait, ne comportant pas une seule illustration, et ne prend pas en compte les questions qui viennent en aval des décisions ainsi prises. Ainsi, derrière sa volonté de rupture affichée avec le paradigme classique de la décision, ce texte partage avec lui une grande partie des hypothèses que tous deux empruntent à la théorie micro-économique : surreprésentation des déterminants matériels dans les pratiques des acteurs, absence de réflexion sur la formation de leurs préférences, abstraction d'un raisonnement largement désocialisé.

Contrastant avec ces approches, l'analyse faite par Haroun Jamous (1969) de la réforme hospitalière française de 1958 fait preuve d'une grande maturité, mais elle est restée longtemps isolée. Elle innove en montrant l'importance du contexte sociohistorique de l'action. Cette réforme n'aurait en effet pu avoir lieu sans la présence d'une nouvelle catégorie de jeunes médecins, d'origine plutôt modeste et qui ne parviennent pas à se faire entendre dans le système tel qu'il existait alors. Ce sont eux qui vont porter la " personnalité charismatique »- deuxième trait de la réforme, emprunté aux catégories de la sociologie weberienne - qui donnera son nom à celle-ci : la réforme Debré. En enracinant son analyse dans des matériaux sociohistoriques concrets, $\mathrm{H}$. Jamous pointe ainsi une limite de ce qui constituait déjà un progrès par rapport aux théories les plus classiques de la décision, à savoir l'analyse en partie systémique de Michel Crozier (1963) qui, écrit-il, ne permet pas de rendre compte du moment de la réforme. Enfin, l'auteur s'appuie sur des entretiens qu'il cite abondamment et qui livrent non seulement des informations factuelles, mais aussi les catégories de pensée de ses réformateurs, comme des valeurs proches du mendesisme. Cette étude socialise donc enfin l'action publique. À partir de matériaux empiriques concrets, en montrant l'importance des trajectoires sociales des acteurs, elle permet d'appréhender ce qui, pour l'approche traditionnelle, n'était qu'une préférence exogène non interrogée et non prise en compte. Pendant des décennies, elle n'aura malheureusement que peu de suites visibles.

8. Cela provient vraisemblablement d'une absence d'informations sur ce qui pourrait leur faire accorder plus ou moins d'importance à tel ou tel problème, autrement dit d'information sur les préférences des agents, qui sont supposées exogènes et dont la formation n'est pas interrogée. 


\section{Un déplacement méthodologique limité}

En tant que science sociale, l'analyse de l'action publique peut pourtant faire flèche épistémologique de tout bois méthodologique. Comme le soulignent $\mathrm{P}$. Bongrand et $\mathrm{P}$. Laborier (2005), un travail aussi ancien que celui de Peter Blau (1955) avait ainsi recours à une palette d'outils diversifiés, allant de l'observation directe aux statistiques en passant par les entretiens. Or, poursuivent les auteurs, les études françaises des années 1990 sont beaucoup moins riches : I'observation n'est quasiment pas pratiquée alors même que des occasions propices s'offrent ; les entretiens sont utilisés la plupart du temps sur un mode restrictif, celui de la collecte rétrospective d'informations, qui repose sur la fragile hypothèse de l'homologie entre discours et pratiques des acteurs. Quant aux méthodes quantitatives, qui ne sont pas mentionnées dans l'article, elles sont vraisemblablement peu mobilisées par les chercheurs français.

Ainsi, l'analyse des politiques publiques semble avoir connu un déplacement méthodologique limité. Ce déplacement existe, comme en témoignent certains textes mentionnés ici. Mais il demeure circonscrit à certains sous-courants de la discipline, ou se trouve sous la forme d'importation en contrebande, c'est-à-dire que certains travaux extérieurs à la discipline au sens strict, relevant par exemple d'autres branches de la science politique ou même de la sociologie, peuvent être relus et assimilés dans une perspective d'analyse de l'action publique. Pour une partie de celle-ci, on se trouve plutôt dans une situation par rapport à laquelle l'usage d'entretiens, fussent-ils purement informatifs et rétrospectifs comme le déploraient $P$. Bongrand et $P$. Laborier, serait déjà une forme de progrès. La deuxième partie de ce texte peut donc être lue comme s'inscrivant dans la continuité des réflexions formulées par ces deux auteurs en suggérant un renouvellement méthodologique.

\section{L'ethnographie : un mode de connaissance propice à rendre compte de l'action publique}

II ne s'agit pas de procéder ici à une présentation exhaustive de ce qu'est l'ethnographie, ni de ses rapports avec les autres sciences sociales ${ }^{9}$. On souhaiterait en revanche en souligner quelques traits qui la rendent intéressante pour notre propos. Puisqu'il s'agit d'une enquête directe par un enquêteur qui doit nouer des relations personnelles avec les personnes du milieu enquêté, il faut préciser A) la position de l'enquêteur, B) les méthodes qu'il utilise, C) son mode d'appréhension des enquêtés.

\section{La position de l'enquêteur : contre l'ethnocentrisme}

Construite notamment en réaction contre l'ethnocentrisme culturel, l'ethnographie est une discipline propice pour parer au risque d'ethnocentrisme de position qui guette tant l'analyse de l'action publique. À ce titre, deux temps peuvent être distingués dans sa démarche :

9. Sur ce point, cf. de manière générale Passeron, 1991, chap.1; Weber, 2009, introduction. 
1) La saisie de la dimension relationnelle des situations. II s'agit de plonger dans le subjectivisme qui fait défaut aux analyses classiques de politiques publiques, qui se donnent pour objectives alors qu'elles ne se sont pas interrogées réflexivement sur leur point de vue.

2) Une fois ceci acquis, il convient de faire varier le point de vue de l'observateur. C'est la combinaison de nombreux points de vue subjectifs et leur synthèse qui permet d'accéder à un plus grand degré d'objectivité.

\section{Objectiver sa propre position}

L'ethnocentrisme de position peut être réduit tout d'abord par l'explicitation de la position de l'observateur. Même si les énoncés scientifiques sont impersonnels $\left(E=M C^{2}\right.$; il existe une certaine reproduction sociale ; etc.), leur découverte est le fait de certaines personnes, dotées de certains instruments. Les objets des sciences sociales - les normes juridiques et morales, l'État, la famille, etc. - concentrant déjà sur eux, comme le notait Durkheim (1895, p. 15), une "réflexion antérieure à la science " du fait que "l'homme ne peut pas vivre au milieu des choses sans s'en faire des idées d'après lesquelles il règle sa conduite ", les découvertes en la matière sont fortement liées aux caractéristiques des chercheurs. Ces caractéristiques doivent donc être explicitées car elles peuvent conditionner les résultats d'une recherche. Par exemple, la mise en évidence de la notion, aujourd'hui reprise dans de multiples travaux, de "travail à côté " par Florence Weber (1989), n’a été possible qu'en raison de certains éléments biographiques : héritière de capital intellectuel à une génération, l'auteur fait état d'un rapport affectif profond à la classe ouvrière, qui a vraisemblablement orienté la direction de ses recherches; ce n'est que grâce à l'insertion locale de sa famille qu'elle a pu surmonter la barrière sociale existant entre elle et les ouvriers enquêtés, confirmant du même coup la prégnance de ces deux facteurs. De manière analogue, Michael Burawoy (1998) préconise d'étendre l'observation à l'enquêteur : partant du constat que l'idéal positiviste d'un observateur neutre raisonnant sur des échantillons parfaitement représentatifs est impossible, il recommande de réintroduire dans les matériaux d'enquête l'inévitable perturbation introduite par celui-ci (" interventions create perturbations that are not noise to be expurgated but music to be appreciated ", p. 14). II montre ainsi, à partir des enquêtes ethnographiques qu'il a effectuées dans l'industrie du cuivre de la Zambie des années 1970, comment se perpétue une domination raciale (color bar) même après la décolonisation officielle, domination qui apparait jusqu'entre lui, professeur blanc, et ses étudiants noirs.

Certes, mettre en avant la singularité des situations d'enquête et des enquêteurs n'est pas sans risques (Olivier de Sardan, 1995, 2000). On peut mentionner parmi eux tout d'abord celui du narcissisme, quand la focale se déplace de l'objet enquêté à l'enquêteur lui-même. Dans ce cas on est en quelque sorte trompé sur la marchandise, qui n'est plus l'objet officiel mais un autre, l'auteur, dont on se serait bien passé. Un autre risque est de quitter le domaine de l'écrit scientifique pour aller vers celui de la littérature, de rechercher dans son texte non la simple vérité mais l'esthétique. Bien sûr, les deux peuvent aller de pair, comme dans certains textes de Lévi-Strauss, et sont même parfois recommandés afin de mieux se faire comprendre (Becker, 2004). Mais l'écrit en sciences sociales ne doit pas chercher à compenser ce qui serait une insuffisance de preuve par des "effets de réel " (Passeron, 1991) 
purement littéraires. Cette dérive tentant de compenser un manque de fond par un supplément de forme caractériserait en particulier certains travaux d'anthropologie culturelle.

Dans ces conditions, le bon usage du "je " en sciences sociales semble devoir obéir à quelques règles simples. Tout d'abord, il ne doit pas être conçu comme une fin de l'enquête en elle-même, mais juste comme un instrument de connaissance. Réfléchissant au moyen de remédier au célèbre paradoxe de l'observateur (Labov, 1972), Olivier Schwartz indiquait ainsi que les matériaux d'enquête, grevés par cette relation, devaient être traités non comme des représentations immédiates d'une réalité naturelle mais comme des effets de la situation d'enquête, des artefacts. "À qui [les enquêtés] croient-ils s'adresser lorsqu'ils parlent à l'ethnographe et que cherchent-ils à "faire" de la relation qui s'instaure avec lui ? " (1993, p. 275). Voilà la question à laquelle il faut avoir répondu préalablement pour pouvoir faire un usage valable des matériaux ainsi récoltés ${ }^{10}$. II faut donc bien commencer par avoir quelques idées sur l'ethnographe en question. Cela permet surtout de bien contrôler que le résultat n'est pas dépendant d'une propriété sociale donnée de l'enquêteur et qu'il aurait été autre avec une autre propriété sociale, autrement dit de vérifier son objectivité, d'aboutir à ce que Pierre Bourdieu (2003) nomme une objectivation participante, laquelle "vise une objectivation du rapport subjectif à l'objet qui, loin d'aboutir à un subjectivisme relativiste et plus ou moins antiscientifique, est une des conditions de l'objectivité scientifique » (p. 44). La question devient dès lors : quelles caractéristiques de l'enquêteur plutôt que d'autres doivent être explicitées?

Des travaux existent, en dehors du champ de l'analyse de l'action publique, qui ont déjà apporté des éléments de réponse à cette question. Par exemple, comme le soulignent nombre de féministes, des terrains identiques ont produit des résultats d'enquête différents selon que l'enquêteur était un homme ou une femme, d'où l'intérêt de travailler finalement en couple (Weiner, 1976 ; Descola, 1994 ; Bertaux, 1996 ; Bertaux-Wiame, 2004). Avec le genre, d'autres variables sociologiques lourdes rendent compte de la possibilité d'avoir accédé à certains résultats : la position et la trajectoire sociale (Mauger, 1991 ; Bourgois, 2001), l'appartenance à une certaine génération et donc aussi à une certaine classe d'âge (Fournier, 2006 ; Lignier, 2008), etc. Dès lors, employer, dans une restitution scientifique, le « je " plutôt que le « il » ou le " elle " (ou pire encore recourir à une trompeuse forme impersonnelle : «il est apparu que... »), n'est ni faire preuve de complaisance envers soi, ni basculer dans l'essayisme littéraire, mais rappeler au lecteur, à condition que le « je » en question soit clairement objectivé, le caractère situé de toute connaissance, particulièrement dans les sciences sociales. En revanche, dans l'étude de l'action publique, cet apport ne semble que très peu mobilisé à ce jour.

\section{Se déplacer au sein de l'objet d'étude}

Après une bonne objectivation de l'instrument d'observation, une autre ressource offerte par l'ethnographie réside dans le déplacement de celui-ci au sein de son objet d'étude. II s'agit d'observer successivement les quatre niveaux d'une action publique que nous avons recensés, sans compter les variations au sein de chacun d'eux suivant les acteurs concernés. Cela permet de se départir de l'ethnocentrisme de position dans lequel on reste inévitablement

10. De manière plus générale, utiliser la position de l'observateur en tant que révélateur du terrain correspond à un courant de recherche important (pour plus de précisions, cf. Schwartz, 1993). 
enfermé si on n'observe qu'un seul d'entre eux, particulièrement celui de l'édiction des normes qui, puisque ces normes sont publiques, se donne particulièrement à voir.

Dans les sciences sociales en général, ce principe de déplacement est déjà couramment mis en œuvre. Dans son ouvrage fondateur, Malinowski (1922) se déplace dans les différentes îles de l'archipel des Trobriand pour saisir leurs populations à la fois dans leurs spécificités, comme leurs systèmes familiaux ou sociaux, et dans ce qu'elles ont en commun, telles la participation aux échanges rituels (kula) et leurs croyances magiques. À l'heure où les analystes des politiques publiques s'interrogent sur l'éclatement toujours plus grand des acteurs et sur l'autonomisation de leurs croyances par rapport à celles de leurs voisins et au socle de leur action réelle, on ne saurait sous-estimer l'intérêt de la comparaison avec cette forme archipellaire.

Plus proche de nous dans le temps et dans l'espace, c'est à nouveau le déplacement d'un observateur dans un certain milieu, celui des rémunérations bancaires, qui permet d'obtenir des résultats novateurs (Godechot, 2006, 2007). L'auteur montre ainsi, en comparant le côté des dirigeants et des théories officielles avec celui des exécutants - en l'occurrence les traders - que la théorie dominante, celle du modèle principal-agent, dont nous avons vu plus haut à quel point elle est justement liée à un ethnocentrisme de position, rend moins bien compte du phénomène des bonus qu'un modèle alternatif, celui du hold-up. Au cœur de l'analyse des politiques publiques, se trouve l'approche d'ethnographie critique définie par V. Dubois (2012), consistant à rompre avec le discours officiel sur la restructuration de l'État social pour montrer au contraire, à partir d'enquêtes au contact direct des bénéficiaires de ces politiques, le tournant coercitif de celles-ci. Enfin, George Marcus (1998) propose une généralisation de la formulation de Malinowski en suggérant de se déplacer au sein non plus seulement de populations, mais aussi (en s'inspirant d'I. Wallerstein) de chaînes de production et de circulation d'objets matériels, comme des monnaies, des œuvres d'art ou des biens manufacturés, ou encore (à la suite de M. Halbwachs et de C. Lévi-Strauss) d'objets immatériels, comme les formes mémorielles, des mythes, et autres figures de la pensée. Dans tous les cas de figure, cela donne une ethnographie multisituée, au sens où elle comporte plusieurs entrées, qui peuvent être socialement très éloignées, entre lesquelles il s'agit de naviguer, et où elle définit son objet par leur réunion.

Relativement utilisé dans les sciences sociales en général, ce principe du déplacement n'est pas encore pleinement mobilisé pour analyser les politiques publiques. II est certes théorisé, de façon différente selon les écoles : approche à la fois "par le haut » et "par le bas " dont il a été question plus haut ${ }^{11}$, reconstitution des "séries causales qui mènent des lieux les plus centraux de l'État jusqu'aux régions les plus déshéritées du monde social » (Bourdieu, 1993), ou encore variation des «échelles de l'observation » (Revel, 1996). D'un point de vue empirique en revanche, ce principe est encore relativement peu mis en œuvre. II faut reconnaître que cette opération présente une certaine difficulté car elle oblige à multiplier les terrains d'enquête et à trouver les liens pertinents entre eux. Ainsi, « établir un lien

11. Ce qui pourrait correspondre aux deux niveaux extrêmes de nos quatre entrées. Cette formulation est cependant imprécise en ce qu'elle omet les deux autres. Elle induit en outre un risque de confusion avec l'échelle sociale: les destinataires d'une action publique où ses acteurs du premier rang ne se situent pas nécessairement « en bas " de la hiérarchie des PCS, comme l'illustre le cas des patients aisés ou celui des médecins. Enfin et surtout, comme on l'a vu, elle fait preuve d'ethnocentrisme de position. 
entre ce qui ressort de l'étude des agents des sociétés HLM et la politique du logement en France, entre le travail du guichetier de la CAF et les réformes des affaires sociales, entre l'universitaire face à ses étudiants et la politique contractuelle, ne va pas de soi " (Musselin, 2005, p. 53-59). De fait, la plupart des travaux existant sur les politiques publiques se cantonnent à un seul des quatre niveaux d'étude. II peut s'agir de celui de l'édiction des normes, selon le biais classique précédemment relevé, y compris quand les intéressés font de l'ethnographie (Cambrosio et al., 1990 ; Latour, 2002). II peut s'agir à l'inverse de celui de leurs destinataires ou des acteurs du premier rang (Stack, 1997 ; Dubois, 1997 ; Weller, 1999 ; Cartier, 2003 ; Siblot, 2006).

Quelques études cependant naviguent entre les différents niveaux d'une action publique. Dans son analyse de la politique migratoire en France de 1945 à 1975, Alexis Spire (2005) expose tant les logiques qui président à sa conception, à partir d'archives ministérielles, que son application par ses acteurs du premier rang, en l'occurrence les guichetiers, à la lumière de leurs trajectoires sociales saisies par des entretiens, et que ses conséquences en termes de délais d'obtention des titres de séjour par les étrangers, destinataires statistiquement objectivés. Émilie Biland (2008) fait jouer à plein le principe de déplacement entre niveaux d'analyse à propos de la politique de recrutement des fonctionnaires territoriaux. Son enquête se déroule aussi bien du côté de la conception des normes, avec des archives et des entretiens auprès des dirigeants impliqués dans cette phase, qu'à celui des acteurs du premier rang, les personnes chargées du recrutement des fonctionnaires territoriaux, et enfin des destinataires, les candidats et agents eux-mêmes. Elle est multisituée également d'un point de vue géographique, puisqu'elle se déroule à la fois dans une collectivité locale - la ville de Tozay -, une institution nationale - le CNFPT - et deux centres départementaux de gestion de la fonction publique territoriale, l'un francilien, l'autre rural, l'ensemble constituant un dispositif d'enquête étoffét ${ }^{12}$.

\section{Utiliser tous les outils des sciences sociales}

Toutes sortes de matériaux sont utilisables et certains sont déjà bien utilisés, comme les archives. Au-delà d'un appel à ne négliger aucun d'entre eux, y compris les statistiques, nous voudrions simplement ici, dans la lignée de $\mathrm{P}$. Bongrand et $\mathrm{P}$. Laborier, suggérer de : 1) recourir à l'observation directe, 2) pratiquer des entretiens non plus simplement informatifs mais ethnographiques.

\section{Participer et observer}

Parmi les méthodes qui s'offrent à l'analyste de l'action publique, l'observation participante est encore largement sous-utilisée ${ }^{13}$. Elle est pourtant, par l'immersion prolongée dans le milieu étudié qu'elle suppose, autrement dit par la socialisation intensive et/ou durable de l'enquêteur aux catégories des enquêtés, un outil singulièrement utile pour dépasser tout

12. Une éclairante synthèse graphique de ce dispositif est disponible (Biland 2008, p. 37).

13. En raison du paradoxe de l'observateur, la participation est toujours première par rapport à l'observation (et même à l'entretien éventuel). C'est pourquoi on trouve également dans la littérature le terme de " participation observante ", la différence entre les deux étant au demeurant ténue (Soulé, 2007). Nous utilisons classiquement ici le terme d' "observation " pour caractériser les situations où l'observateur ne joue que ce rôle, et celui d'« observation participante » quand il joue en outre un autre rôle. 
ethnocentrisme, de position ou autre, et pour remédier au fameux paradoxe de l'observateur. Malinowski (1922, p. 64-65) l'explique clairement :

"Parce qu'ils me voyaient tout le temps parmi eux, les indigènes n'étaient plus intrigués, inquiets ou gênés de ma présence; dès lors je cessais d'être un élément perturbateur dans la vie tribale que j'étudiais, je ne faussais plus tout du fait de mon approche [...]. En réalité, comme ils savaient que je fourrerais mon nez partout, même là où un indigène bien éduqué ne songerait pas à s'immiscer, ils finissaient par me regarder comme une part et un élément de leur existence, un mal ou un ennui nécessaires, atténués par les distributions de tabac. ”

L'observation, en outre, révèle des choses qui ne sont ni écrites, ni dites, et qui ne peuvent donc être saisies par les autres techniques d'enquête.

"Ces choses, même si elles sont cristallisées et bien établies, ne sont nulle part formulées. II s'agit d'un code de lois non écrit et non explicite, et l'ensemble de la tradition tribale comme l'ensemble de la structure sociale se trouvent enfermés dans le plus insaisissable des matériaux : l'être humain (...). Il serait vain d'essayer d'interroger un indigène en termes sociologiques abstraits " (ibid., p. 68) ${ }^{14}$.

Les choses qui ne sont pas formulées consciemment transparaissent tout d'abord au niveau infra-individuel, celui des corps. Ainsi, en éprouvant directement les contraintes qui sont posées aux enquêtés (Linhart, 1978), voire en acquérant progressivement l'habitus propre au milieu étudié (Wacquant, 2000 ; Sorignet, 2010), l'enquêteur accède à une compréhension beaucoup plus riche et fine de ce qui se joue. Symétriquement, l'observation permet aussi de saisir des éléments qui échappent aux consciences individuelles parce qu'ils les dépassent, comme les évolutions insensibles de certaines données au cours du temps. Ainsi peut-on mettre en lumière la trajectoire de cas cliniques au sein de services de soin, qui n'apparaissent que par le constat de variations du contenu des interactions observées au cours du temps et qui demeurent invisibles si on se limite à opérer des entretiens (Paillet, 2007).

En ce qui concerne plus particulièrement les politiques publiques, l'observation est particulièrement propice à la mise en lumière des jeux avec la règle ou entre les règles. II est reconnu que la mise en œuvre d'une action publique peut être menacée par l'écart existant entre la règle de droit qui lui sert de vecteur principal et les pratiques effectives de ceux chargés de cette mise en œuvre, que ces pratiques se présentent clairement comme des mises à distance, des passe-droits, des transgressions (Bourdieu, 1990), ou comme un aménagement de la règle de droit primaire sous forme de normes secondaires, d'interprétation, de négociation ou de règlement des conflits (Lascoumes, 1990). Mais au-delà de ce qui apparait largement comme une querelle d'écoles, les jeux avec les règles sont révélés par l'observation concrète des agents du premier rang, comme des agents des Caisses d'allocations familiales en visite à domicile (Dubois, Dulong, 2003) ou au guichet (Dubois, 1997) : la règle constitue pour eux à la fois une contrainte possible à transgresser sous certaines conditions et une ressource pour l'action face aux usagers; ce jeu permet d'imposer la politique en question à ses destinataires. Ainsi, seule l'observation

14. Si le domaine d'application de telles réflexions a longtemps été cantonné aux terrains exotiques, la fin du " grand partage " entre disciplines (Latour, 1988 ; Lenclud, 1992) fait qu'il est désormais généralisé. 
ethnographique permet de mettre en lumière certaines pratiques essentielles à la compréhension des politiques publiques, précisément parce qu'elles ne sont objectivées dans nul texte ou discours légitime.

\section{Des entretiens, mais ethnographiques}

Après l'observation, les entretiens peuvent à leur tour être mobilisés pour l'analyse des politiques publiques, mais selon un mode ethnographique encore peu pratiqué aujourd'hui. II s'agit non de prendre les discours des enquêtés au pied de la lettre, mais de les considérer comme des symptômes d'autre chose qu'eux, notamment de leurs catégories de pensée. C'est ce type de critique que formule Sylvain Laurens (2007) quand il note, à propos des entretiens avec des "imposants ", que ceux-ci sont une participation avant que d'être des entretiens. On peut généraliser: dans l'ordre de mobilisation des méthodes en sciences sociales, la participation, éventuellement observante, vient avant l'entretien ; l'entretien n'en est qu'une modalité, dans le sens où il s'inscrit dans la participation de l'enquêteur à une certaine situation sociale qui lui préexiste nécessairement. Tout entretien, avec quelque personne que ce soit, délivre certes des informations, mais le rapport de celles-ci avec la "réalité » dépend de la situation d'entretien elle-même, c'est-à-dire de la personne à qui l'enquêté pense s'adresser quand il parle à l'enquêteur. À la suite de F. Weber (2009, p. 65), on peut faire un parallèle entre cette démarche et celle d'un historien comme Marc Bloch (1946) :

"S'en tenir aux réponses volontaires faites à des questions directes, ce serait commettre la même erreur que s'en tenir à la lecture au premier degré de la vie des saints [allusion à un texte de Simiand auquel se réfère Bloch] : y chercher des renseignements sur "les pieux personnages dont elles prétendent retracer le destin". Transformer les entretiens en véritables sources, c'est considérer les enquêtés comme des "témoins malgré eux" et non comme des porte-parole. "

Par exemple, tel magistrat, en exprimant en entretien le contraste entre les idéaux de justice dans lesquels, issu d'un milieu catholique social, il a grandi, et le fonctionnement pratique actuel de l'institution judiciaire, ne livre que des informations historiques très vagues sur sa propre histoire et sur celle de son employeur, mais révèle au contraire, en tant que "reproche vivant ", l'incapacité de cette institution à réaliser ce pour quoi elle est conçue (Lenoir, 1993).

\section{Ne pas ignorer certaines dimensions essentielles des acteurs}

Après les variations de la position de l'observateur et l'utilisation de tous les outils des sciences sociales qu'elle autorise, un troisième apport de l'ethnographie à l'analyse de l'action publique réside dans la prise en compte des différentes appartenances sociales des acteurs. Ce terme désigne, plus largement que «l'identité ", l'insertion, volontaire ou non, dans des collectifs qui informent nos manières de sentir, de penser et d'agir, comme le groupe professionnel, la classe sociale, la famille, la nation, etc. (Avanza, Laferté, 2005 ; Weber, 2005). Les enquêteurs ont également diversifié les types d'appartenances qu'ils prennent en compte. Aux débuts de l'ethnographie, ils se référaient volontiers de manière exclusive à certaines formes de totalisations prises dans une liste limitative contenant principalement l'ethnie, la culture et la classe sociale. L'imbrication de ces réalités sociales entre elles et l'existence d'autres cadres causatifs, comme le genre ou la génération, les conduit 
aujourd'hui à reconnaître la validité concomitante de différentes formes d'appartenances, à pratiquer une ethnographie désormais « combinatoire » (Baszanger, Dodier, 1997) ou « multiintégrative » (Weber, 2003).

Or, l'analyse de l'action publique raisonne classiquement sur les seules appartenances professionnelles, même quand elles sont saisies d'une manière très fine grâce par exemple à une sociologie interactionniste inspirée d'auteurs tels qu'Erwin Goffman (Weller, 1999 ; Warin, 2002). Cette relative apesanteur sociale fait comme si des variables aussi lourdes que la trajectoire sociale, le genre, la génération, l'appartenance à des minorités, la socialisation politique, etc., n'avaient aucune influence sur les façons de travailler ${ }^{15}$. Elle semble faire siens certains aspects de l'idéal weberien du fonctionnaire, qui dans le cadre de ses fonctions ne subirait aucune influence extérieure, parvenant ainsi à les séparer totalement de sa « vie privée ».

Ces approches manquent cependant de réalisme. Si, comme le montrent les sociologues des professions en général (notamment Freidson, 1970), ce qui est fait au travail se comprend entre autres par les conditions de son accomplissement, le " présent » des agents ne pouvant être oublié au profit de leur seul "passé ", les cadres plus généraux de cet accomplissement, ainsi que le passé en tant que moment de socialisations dont les effets perdurent, contribuent aussi à cette compréhension. La mobilisation de ces cadres d'analyse permet de jeter une lumière vive sur nombre de métiers (Avril et al., 2010). Ce qui vaut pour le travail en général s'applique aussi à la production des politiques publiques. Les agents par qui elles existent se caractérisent ainsi par leurs "deux corps " (Kantorowicz, 1957 ; Dubois, 1997 ; Belorgey, 2011a) : le corps officiel, celui de leur fonction publique, et leur corps en chair et en os, c'est-à-dire leur être social, qui contribuent de concert à la production de la politique publique concernée. De manière analogue, les assistantes sociales en charge de la petite enfance font plus ou moins de signalements d'enfants "en danger " selon qu'elles se réfèrent plutôt aux avis de leurs chefs ou de leurs collègues et selon leur trajectoire sociale, celles d'origine populaire qui ont en plus connu des expériences sociales atypiques adoptant facilement un regard relativiste et compréhensif à l'égard des familles populaires (Serre, 2001, 2009, p. 141-260, 299).

Un exemple d'ethnographie combinant appartenances de classe, de génération et de genre est fourni dans un domaine aux confins de l'action publique, celui de la distribution du courrier (Cartier, 2003). Trois générations de facteurs entretiennent en effet des rapports différents à cette activité. Ceux des Trente Glorieuses sont surtout des hommes d'origine ouvrière. Heureux de pouvoir rester sur une "tournée " qui leur permet de développer une sociabilité locale, ils aiment "rendre service " et "faciliter la vie des gens". Les facteurs recrutés entre 1974 et 1983, plus souvent des femmes, sont issus de milieux plus variés, et caractérisés par un investissement antérieur dans les sphères politique ou religieuse. Pour elles, le "service public " a un sens beaucoup plus fort que pour la génération d'avant, particulièrement quand celui-ci est remis en cause à partir du tournant de 1983. Enfin, à partir de cette année, la tendance jusque-là croissante des effectifs s'inverse, préludant au

15. Pour une revue de la littérature plus complète sur ces questions, cf. Dubois, 1997, p. 16-18 et Avril, Cartier, Siblot 2005. 
changement de statut de La Poste (1990) et à l'injonction envoyée aux facteurs par leur direction de devenir des commerciaux. Les relations avec les usagers deviennent tendues et la qualité du service postal se dégrade (p. 237-256).

Au-delà du principe de multi-appartenance des personnes, quelles variables peut-on négliger et lesquelles faut-il prendre en compte pour les caractériser dans le cadre d'une action publique ? II faut utiliser tout d'abord les variables classiques de l'ethnographie. On s'intéressera donc à la trajectoire sociale des personnes, à leur génération, à leur genre, à leur race (Hughes, 1946 ; Fassin, Fassin, 2006) ${ }^{16}$. On pourra étudier les effets de ces facteurs séparément ou en explorant leurs intersections de manière combinée (comme par exemple Kergoat et al., 1992 ; Chauncey, 1994 ; Bessière, 2003). Nombre de ces variables sont déjà mobilisées en science politique, par exemple dans l'étude de l'action collective (pour n'en citer qu'un: Siméant, 1998). II serait étonnant que, passant de "collective " à " publique ", "l'action " change à ce point de nature, que ce qui était déterminant devienne soudain inopérant. S'il faut voir une spécificité dans l'action publique, c'est peut-être dans la plus grande structuration des contraintes organisationnelles ou institutionnelles qui la caractérisent. Mais puisque toute action publique est aussi collective, des traits de celle-ci doivent se retrouver dans celle-là, un peu comme la légitimité traditionnelle, chez Max Weber, se comprend comme une routinisation (veralltäglichung) de la légitimité charismatique qui l'a fondée initialement. À ce titre, certains travaux présentent une combinaison particulièrement intéressante, en montrant à la fois le poids du "passé ", en l'occurrence que le mouvement de grève des loyers dans les foyers Sonacotra dans les années 1970 a réinvesti dans la lutte des savoir-faire acquis par ses principaux militants dans les luttes anticoloniales, et l'importance du "présent ", sous la forme d'une subversion de l'institution existante des logements, dont les caractéristiques architecturales sont reconverties en espaces propices à la mobilisation (Hmed, 2007).

II faut ensuite accorder toute leur importance aux variables spécifiques à l'action publique. On y trouve le rapport des acteurs à leur travail et leur place dans un système professionnel ou organisationnel (pour un exemple récent, cf. Castel, 2005). On y trouve également les appartenances institutionnelles, l'institution se définissant par la rencontre dynamique entre ce qui est institué (aussi bien au sens strict, par exemple le Conseil d'État, qu'au sens large, par exemple le droit public) et les investissements des membres qui la font exister d'une certaine façon (Offerlé, Lagroye, 2010). On y trouve enfin leur rapport à l'action publique en question, qui peut être dans les cas extrêmes favorable, quand il s'agit des auteurs d'une politique publique donnée, ou défavorable, pour les opposants déclarés à cette politique, avec toutes les situations intermédiaires d'appropriations variées (cf. infra). Ainsi, la démarche ethnographique peut aussi être enrichie par son ouverture à des formes d'appartenances autres que celles issues de la socialisation primaire, qui historiquement constitue son terrain d'élection favori. II s'agit d'augmenter le nombre de combinaisons d'une ethnographie combinatoire, ou d'intégrer plus de dimensions dans le cadre d'une ethnographie multi-intégrative.

16. Au sens bien sûr non d'une essence, connotation qui fait que ce terme est rarement employé en sciences sociales, mais d'un rapport social, par exemple entre deux groupes qui se définissent mutuellement en termes raciaux. 


\section{L'ethnographie de l'action publique hospitalière}

Nous proposons à présent d'illustrer ce qui précède - les insuffisances de l'analyse de l'action publique dans son état actuel et les remèdes qu'elles appellent ; la méthode ethnographique appliquée à l'analyse de l'action publique - par un cas d'espèce : l'analyse de l'action dans le domaine hospitalier en France dans les années 2000, et plus particulièrement sur une partie de ce qui a été nommé le « plan Hôpital 2007 ». Nous allons exposer quelques méthodes ethnographiques mobilisées à cette occasion puis quelques résultats auxquels elles ont permis d'aboutir.

\section{Méthodes et « ficelles »}

L'enquête a eu recours à différentes entrées sur le terrain, qui ont conditionné d'abord ce qui était montré spontanément à l'enquêteur, puis les sources auxquelles celui-ci a pu dans un second temps accéder de son propre chef.

\section{Parcourir la configuration des acteurs : une ethnographie multisituée}

Conformément à ce qui est exposé supra, j'ai recours à plusieurs entrées sur le terrain et me déplace dans la configuration des acteurs ${ }^{17}$. Celle-ci est doublement dispersée. Elle l'est d'abord verticalement, puisque l'on y retrouve les quatre niveaux d'étude d'une action publique : édiction des normes (par exemple le ministère de la Santé), intermédiaires (directeurs d'hôpital, chefs de service, etc.), acteurs du premier rang (soignants) et destinataires (patients). Cette action est aussi dispersée géographiquement, car elle a l'ambition de couvrir tout le territoire français. Diplômé de l'IEP Paris, je rentre le plus facilement par le milieu des directeurs d'hôpital (la majorité d'entre eux ont à ce moment cette formation). Mon premier terrain est l'administration d'un établissement, celui de Migny ${ }^{18}$. À son tour, celle-ci me met en contact avec différents services de soin et notamment celui qui va devenir un de mes principaux terrains d'enquête, son service d'urgences. Ces services sont intéressants en ce qu'ils révèlent des tensions propres à l'institution hospitalière en général (Dodier, Camus, 1997). Pendant les deux années où j'y séjourne par intermittence, j’y joue le rôle d'un étudiant de passage, ce qui me permet de recueillir observations et entretiens approfondis auprès de ses soignants. J'objective également ce que font les patients, notamment par une étude en sociologie de la santé sur les patients d'un réseau ville-hôpital œuvrant dans la même zone (Belorgey, 2009).

L'autre grande entrée se situe au niveau de la conception de la politique. Grâce aux connaissances acquises précédemment, je trouve une alliance du côté d'un organisme investi à la fois dans le conseil dans le secteur de la santé et dans le champ académique, le Centre d'intervention en gestion (CIG). Celui-ci me fait à son tour accéder à l'Agence d'audit des établissements de santé (AAES, rattachée au ministère de la Santé), qui s'appuie sur son expertise, et qui apparaît rétrospectivement comme étant aussi un de mes principaux terrains. Quasiment assimilé à un membre de ce prestigieux centre de recherche, je bénéficie à l'AAES d'une grande liberté de circulation. J'observe alors, parfois de manière participante, les auditeurs de l'AAES au contact des services de soin. Cela me permet aussi de mieux

17. Cette troisième partie engageant les caractéristiques de l'enquêteur, elle est écrite en utilisant le « je ».

18. Les noms de lieux, de personnes et d'institutions sont anonymisés. 
objectiver ces intermédiaires, qui jusqu'alors n'avaient pas accepté davantage que des entretiens, refusant au nom de la "difficulté à la faire comprendre au client " la présence d'un sociologue lors de leurs missions. Arrivant cette fois à leur contact par le niveau de la conception, je me trouve du côté du «client », ce qui facilite beaucoup les choses.

Mes terrains liés à cette entrée ne se développent pas selon une logique de monographie de services hospitaliers, mais comme une monographie de l'AAES et de ses consultants, qui engloberait nécessairement leurs relations avec les soignants. La dispersion temporelle et géographique de leur action modèle alors celle de l'enquête. Par rapport au terrain hospitalier approfondi de Migny, j'en réalise de plus légers. C'est ainsi que j'arrive à Maubourg. Je rencontre Francis, le chef du service d'urgences de cet établissement, à l'occasion des réunions qui ont lieu à l'agence. II fait bon accueil au jeune adjoint des membres du CIG qu'il voit en moi, et qui propose de passer dans les établissements, gratuitement, beaucoup plus de temps que les consultants. Je me rends donc à Maubourg, d'abord comme auxiliaire du consultant qui en est responsable, puis plus longuement de manière plus autonome. Le contraste entre les villes de Migny et de Maubourg, et entre leurs services d'urgence, devient rapidement un élément essentiel de l'enquête. À ces deux entrées principales s'ajoutent des entrées ponctuelles, par interconnaissance, à différents niveaux. Quand je retombe sur l'une d'entre elles à partir d'une autre, cela signifie pour moi l'épuisement des potentialités d'inédit d'un terrain, autrement dit que j'ai atteint dans l'enquête ethnographique une relative saturation (Schwartz, 1993): par exemple quand je vois arriver à Migny un auditeur que je n'ai jamais rencontré auparavant, mais qui utilise des méthodes de travail en tout point semblables à celles que j'ai vu pratiquer dans le cadre de l'AAES, et pratiqué parfois moi-même. Cette diversité de terrains n'est cependant pas un désordre; elle est même en soi porteuse d'informations, comme le montre leur cartographie (cf. figure 1).

Ce plan n'est pas un organigramme hiérarchique. Les flèches correspondent à des liens non de subordination, mais de mise en relation. Autrement dit, elles indiquent par quelle personne ou institution j'ai eu connaissance de la suivante. De même, les institutions enquêtées ne sont pas présentées dans leur intégralité : par exemple CHU8 comporte bien sûr aussi des services administratifs, mais ils sont demeurés hors du champ de l'enquête. Cette carte n'est pas non plus un schéma des appartenances sociales des personnes: elle ne replace celles-ci que dans leur environnement professionnel. En revanche, elle restitue pleinement le terrain de l'enquête. Elle le fait tout d'abord au regard des différentes entrées qui viennent d'être décrites. En bas à gauche, le grand cartouche montre l'hôpital de Migny et les flèches qui en montent, les endroits auxquels j'ai pu accéder à partir de mon insertion dans cet établissement. En haut à droite se trouve l'AAES, à laquelle j'ai accédé par le CIG. On y voit les trois "chantiers " auxquels j'ai participé en son sein puis, à partir de chacun d'eux, les flèches qui descendent d'abord vers les cabinets de conseil travaillant dans chacun de ces chantiers, ensuite vers les hôpitaux dont chaque cabinet a la responsabilité. On vérifie donc le principe d'appauvrissement de la connaissance au fur et à mesure que le regard observe des objets plus éloignés (Lévi-Strauss, 1990) : autant un établissement comme Migny, qui a formé une voie d'entrée, est relativement détaillé, avec par exemple aux urgences une trentaine de soignants, l'ensemble de ces personnes ayant fait l'objet d'observations et d'entretiens approfondis, autant un autre comme CHU7, où je n'ai fait que me 
Figure 1 : une enquête multisituée

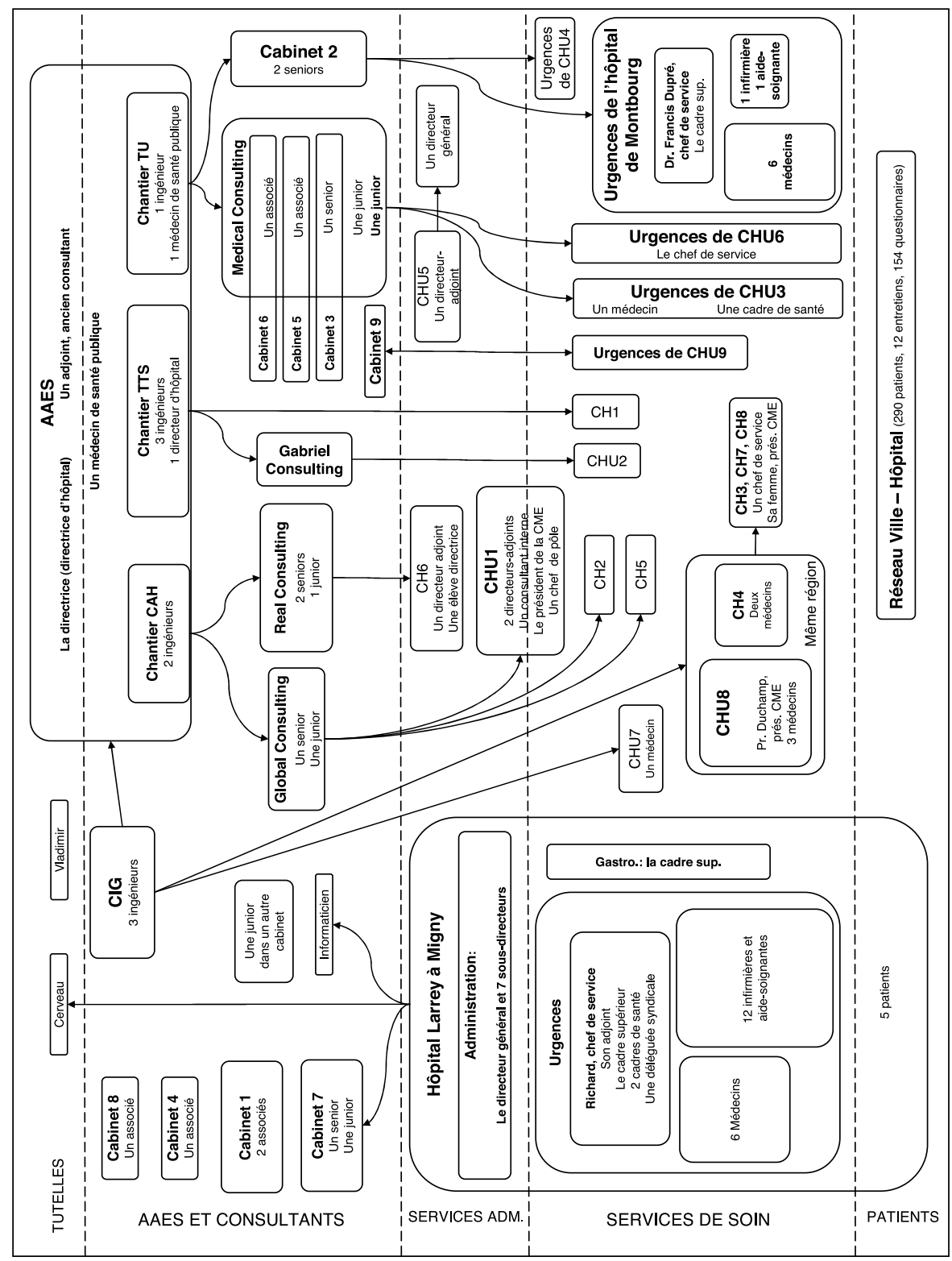


rendre pour une journée avec le CIG, est simplifié à l'extrême, avec juste un médecin ayant fait l'objet d'un entretien, essentiellement informatif. Ainsi, là où il n'y a pour des consultants que des médecins, des infirmières et quelques autres catégories professionnelles, l'implantation longue et directe permet d'accéder aux autres appartenances.

\section{Endosser différents rôles}

Ces différentes entrées me conduisent à endosser, en second lieu, différents rôles socialement définis. Arrivant à l'AAES aux côtés des membres du CIG, j'y suis perçu avant tout comme leur étudiant, ma discipline de rattachement apparaissant secondaire. Au demeurant, la sociologie est largement assimilée dans l'enceinte de l'agence à la sociologie des organisations, et celle-ci à une branche auxiliaire de la gestion. J'accède ainsi, au-delà des discours de " communication » à destination des soignants, aux coulisses de l'agence. Mon objet de recherche initial portait d'ailleurs exclusivement sur ce qui se passait dans les hôpitaux ; l'AAES n'y est rentrée que peu à peu ; comme tant d'ethnographes avant moi, je me suis laissé conduire par le terrain. Arrivant enfin à Maubourg en quelque sorte dans les fourgons de cette agence et de ses consultants, j'y suis perçu initialement comme devant, comme eux, "aider » les soignants à être "plus efficients ».

Autant le rôle que j'endosse à l'AAES et à Maubourg, informé par mon entrée au niveau de la conception des réformes, me conduit à être perçu comme du côté des réformateurs et me permet d'investiguer ce milieu, autant celui qui m'est assigné à Migny, aux côtés des acteurs du premier (et subsidiairement du second) rang de la politique hospitalière, est diamétralement opposé. Le chef des urgences de Migny ne semble en effet m'avoir autorisé à enquêter chez lui que dans l'espoir que je témoigne de la difficulté de la tâche des soignants en ces temps de moyens "rationnés ». Dans chacun de ces services, l'attitude du chef est en outre aussi largement celle des principaux informateurs qui se présentent spontanément à moi où qui se montrent le plus accessibles. II y a donc fort à parier que, si je n'avais étudié que l'un de ces services, j'aurais considéré comme positionnement des soignants face aux réformes en général ce qui n'est en fait qu'un possible parmi d'autres.

On voit aussi également apparaitre à cette occasion l'importance de la variable de positionnement par rapport à la politique étudiée, en l'occurrence appliquée à l'enquêteur luimême. En effet, endossant des rôles différents selon le type d'entrée dont je dispose, je suis aussi destinataire à cette occasion d'informations de nature différente. Quand j'arrive dans les hôpitaux en tant que consultant, par exemple à CHU3 pour le compte de Medical Consulting, les soignants me montrent d'eux un visage tout à fait favorable aux réformes, par exemple en reprenant les mots d'ordre de l'AAES d'une façon qui suggère leur adhésion sincère à eux ${ }^{19}$. Inversement, quand je suis perçu comme sociologue, aux urgences de Larrey, les soignants me dévoilent leurs stratégies de capture des auditeurs : " II suffit que tu saches ce qu'ils vont regarder, et tu fais attention à ça quand ils sont là ${ }^{20}$. »

\section{Accéder à des données complémentaires}

Si le fait de jouer des rôles sociaux distincts rend destinataire des informations différentes directement attachées à la tenue de ces rôles, cela permet également, dans un second

19. Urgences de CHU3, juin 2005.

20. Urgences de Larrey, mars 2005. 
temps, d'accéder de son propre chef à des données plus diversifiées. Ainsi, à Migny, les médecins ne me communiquent pas les données informatiques qui m'auraient permis de caractériser individuellement leurs pratiques professionnelles, selon une démarche classique de refus de laisser les profanes enquêter sur des pratiques médicales. Ces informations, perdues quelque part dans un fouillis de bases de données, existent davantage comme sous-produit de l'activité de soin qu'à d'autres fins et ne sont pas utilisées. Une entrée uniquement par les acteurs du premier rang de la politique ne m'aurait donc pas permis d'accéder à ces données. À Maubourg en revanche, elles font l'objet d'une exploitation systématique de la part de Francis, selon qui ce ne serait que grâce à ce genre d'instruments que certains médecins parviendraient à "se bouger les fesses ". Ces données statistiques existent donc et me sont accessibles, ce qui me permet de les utiliser, mais dans un sens différent : pour tester des hypothèses inductivement forgées au contact de l'ensemble des acteurs de la configuration. C'est donc grâce à la réunion de données obtenues par l'entrée au niveau normatif et de leur analyse à la lumière également des catégories de pensée en vigueur du côté des acteurs du premier rang et des destinataires de la politique, qu'il a finalement été possible de faire progresser l'enquête. Ni ceux qui utilisent habituellement ces données, comme Francis, ni ceux qui refusent par principe d'y recourir, comme les soignants de Migny, n'avaient apparemment pensé à un résultat tel que celui que j'ai finalement obtenu (cf. infra). Les premiers parce que les indicateurs qu'ils utilisent déjà leur permettent, comme ils le souhaitent, de différencier les pratiques médicales selon leur " efficience " (c'est-à-dire, dans une perspective taylorienne, de les inciter à augmenter les cadences de soin). Les seconds parce qu'ils ne trouvent leur défense contre cette attaque que dans un refus général de laisser des profanes établir des distinctions entre pratiques médicales. Des entrées diversifiées étaient donc bien nécessaires pour obtenir de nouveaux résultats.

\section{Quelques exemples de résultats : la politique hospitalière en actes}

\section{Une appropriation socialement modulée}

L'enquête aboutit finalement à plusieurs types de résultats. Le premier d'entre eux concerne les canaux par lesquels cette politique est ou non appropriée par ses acteurs du premier et du second rang. En ce qui concerne les intermédiaires, leur appartenance aux classes moyennes les rend, comme les cadres (Boltanski, 1982), fondamentalement ambivalents. Leurs positions professionnelles peuvent cependant favoriser des mécanismes de conversion, soit pour défendre leur service, parfois au détriment des autres, soit en tant qu'agents privés dépendant de leur commanditaire. À l'inverse, ceux qui n'ont rien à craindre (par exemple, des fonctionnaires statutaires) et qui désapprouvent les normes nouvelles peuvent résister à leur imposition. Les trajectoires professionnelles, quant à elles, rendent compte d'une partition entre vrais croyants, généralistes de formation arrivés par la suite dans le monde de la santé, et faux croyants, au parcours centripète par rapport à ce monde, et qui développent des formes de double conscience. Enfin, la reconstitution précise de leurs trajectoires sociales au sens large montre comment les socialisations antérieures de certains peuvent les conduire à servir les nouvelles normes (par exemple, par la reconversion d'habitus militants) ou à y résister (par exemple, quand elles contrarient une socialisation familiale et associative tournée vers le service aux autres). 
Les logiques qui président aux appropriations soignantes des réformes sont légèrement différentes. La position dans la division du travail hospitalier joue à cet égard un rôle-clé, puisque si leur position dominante dans cette institution comme dans la société en général autorise aux médecins une certaine indifférence face aux logiques gestionnaires, les métiers que l'usage indigène nomme " paramédicaux " (notamment infirmières et aides-soignantes) sont à la fois plus exposés dans leur pratique et disposent de moins de ressources pour faire face aux injonctions normatives relayées par leur hiérarchie. Au-delà de ces appartenances purement professionnelles, l'appropriation éventuelle des logiques managériales par les soignants est fortement conditionnée par leur position dans la configuration établis/marginaux, que l'on retrouve de manière analogue à celle observée entre anciens et nouveaux arrivants dans une petite ville de banlieue (Elias, Scotson, 1965 ; Belorgey, 2010, chap. 7).

\section{L'influence du contexte local}

Un autre facteur de l'appropriation éventuelle de la nouvelle politique par les soignants réside dans les facteurs locaux, c'est-à-dire ceux relatifs à l'histoire des hôpitaux, ainsi qu'à leur position dans le système de soin, comme l'illustre la comparaison des services d'urgence des hôpitaux de Migny et de Maubourg.

À Migny, cette politique pénètre difficilement. Non seulement l'établissement ne participe pas directement aux travaux de l'AAES, mais de plus son histoire récente est tissée de conflits entre les soignants et ceux qui occupent une place intermédiaire entre eux et les concepteurs de cette politique, en l'occurrence la direction de l'hôpital. À la suite d'une première grève des soignants demandant plus de moyens en personnel, la direction tente de promouvoir un audit extérieur. II s'agit pour elle de montrer que si les soignants rencontrent des problèmes dans leur travail, ce serait faute non d'effectifs, mais d'une bonne organisation de leur part, voire de " performance " de la part de certains d'entre eux en particulier. Cet audit bénéficie d'un a priori assez favorable de la part des soignants : la moitié d'entre eux déclarent, dans un questionnaire que je leur fais passer, qu'il aurait avant tout pour objectif d'améliorer le fonctionnement du service. II ne parvient cependant pas à inverser le contexte conflictuel dans lequel il s'inscrit, comme le montre la reprise de la grève quelque temps plus tard, toujours sur des questions de moyens, alors qu'il est censé avoir apporté une réponse pertinente aux problèmes du service et donc prévenu tout nouveau conflit.

La situation à Maubourg est très différente. Le chef de service, Francis, est depuis de nombreuses années un partisan de la nouvelle politique. II participe activement aux chantiers de l'AAES, où il fait figure de " bon élève ". " Ça fait longtemps qu'on réfléchit à l'organisation, I'AAES n'est qu'une étape là-dedans " ${ }^{21}$, explique-t-il. Par ailleurs, le mode de résolution des conflits au travail est plus pacifique à Maubourg qu'à Migny. Comme le résume involontai-

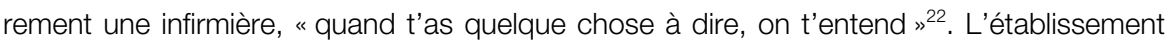
est faiblement syndiqué et les grèves y sont rares. Un audit, d'inspiration très proche de celui de Migny, mais diligenté par l'AAES, a également lieu à Maubourg. II obtient beaucoup plus de résultats. Ses actions reprennent largement les étapes actuelles du travail que Francis mène depuis longtemps, les soignants ne faisant d'ailleurs pas très bien la différence entre

21. Discussion informelle au déjeuner, en marge d'une réunion à l'AAES, novembre 2005.

22. Entretien, novembre 2006. 
les deux. Ainsi, la nouvelle politique, qui s'incarne localement par les réorganisations promues par les audits, semble pénétrer beaucoup plus facilement à Maubourg qu'à Migny.

Or, cette différence de réception est due principalement à des différences de situation économique et sociale. Migny est une "banlieue rouge" (Fourcaut, 1992). Par rapport aux moyennes régionale et nationale, les ouvriers et employés y sont surreprésentés - à elles seules, ces deux catégories forment presque les trois quarts de la population active. Le taux de chômage particulièrement élevé de la ville reflète la fragilité de la situation économique de ses habitants : $21 \%$ en 1999, contre 12,9\% en moyenne nationale. L'hôpital est à l'image de sa commune. Son histoire se caractérise depuis sa fondation au début du $x x^{e}$ siècle par une tension constante au sujet de ses moyens de fonctionnement, entre d'une part ses soignants, parfois même directement ses patients, et d'autre part ses financeurs publics.

Maubourg est une ville beaucoup plus importante, qui a connu une activité économique autonome importante au cours du xxe siècle, notamment industrielle, même si cette dernière est aujourd'hui déclinante. La composition sociale de sa population se situe à peu près dans la moyenne nationale. L'historique officiel de l'établissement fait remonter sa fondation au XIV siècle, et des traces tangibles d'implantation sont visibles en centre-ville au cours du XIXe siècle. II est alors financé par la charité des grandes familles industrielles de la région. L'hôpital bénéficie aujourd'hui de crédits importants et les urgences se financent en outre quelques équipements par des contrats passés avec des laboratoires privés.

Ces données générales se traduisent plus précisément dans un rapport entre l'offre et la demande adressée aux services de soin au niveau départemental beaucoup plus favorable à Maubourg qu'à Migny. La plupart des indicateurs de précarité sociale, qui ont des répercussions sanitaires, sont inférieurs à la moyenne nationale dans le département de Maubourg, tandis qu'ils y sont supérieurs dans celui de Migny. Symétriquement, la plupart des indicateurs d'équipements sanitaires sont supérieurs à la moyenne nationale dans le département de Maubourg, tandis qu'ils lui sont inférieurs dans le département de Migny. On se trouve donc face à une double inégalité : c'est au lieu qui en a le plus besoin que les équipements font le plus défaut, et réciproquement c'est là où la demande est la moins forte qu'on en trouve le plus. L'histoire mouvementée de Migny, notamment ses grèves à répétition, et le calme relatif de Maubourg, illustré par la faiblesse des mouvements revendicatifs de ses soignants, s'expliquent ainsi essentiellement par le rapport entre l'offre et la demande de soin au niveau local. Cette influence concrète du contexte local a été mise au jour grâce à une ethnographie comparée.

\section{Les effets sociaux d'une action publique}

M. Lipsky avance à plusieurs reprises (1980, not. p. 159-172) que les pratiques de contrôle des street-level bureaucrats "sont susceptibles de" (may) dégrader la qualité de leurs prestations, mais sans jamais en fournir de preuve empirique, même de seconde main. Il avance entre autres explications le fait que la baisse éventuelle de la qualité du service serait difficile à mesurer. Or, le recours à l'ethnographie multisituée permet à la fois de définir une mesure opérationnelle des variations dans la qualité et de tester cette affirmation, qui se révèle effectivement confirmée. 
Davantage adoptée par les soignants de Maubourg que par ceux de Migny, la nouvelle politique donne aussi à voir le plus facilement ses effets sociaux dans cet hôpital. C'est aussi celui où j'ai pu accéder à des bases de données chiffrées (cf. supra). Une analyse de seconde main de ces données montre que la nouvelle politique entraîne une hausse du taux de retour des patients à Maubourg : plus les patients sont soignés conformément aux normes nouvelles (réduction du temps qui leur est consacré), plus certes ils le sont de manière apparemment « efficiente ", c'est-à-dire qu'ils passent moins de temps dans le service, mais plus aussi ils courent le risque de devoir revenir après leur sortie pour de nouveaux soins, voire de revenir trop tard pour être soignés (Belorgey, 2011b). Par ailleurs, les soignants qui agissent le plus conformément aux incitations réformatrices sont les " marginaux " du service au sens d'Elias et Scotson, et ceux qui y sont en pratique les plus réfractaires ses "établis ", ce qui confirme l'importance de la prise en compte des appartenances sociales au sens large des intéressés.

\section{Conclusion}

L'ethnographie offre des outils utiles pour l'analyse de l'action publique. Elle permet, grâce au déplacement de l'enquêteur dans la configuration des personnes qui concourent à une action publique donnée, de dépasser l'ethnocentrisme de position qui caractérise encore nombre de travaux de la discipline. Son intégration des différentes appartenances observables des personnes autorise le dépassement du réductionnisme économique et laisse ouverte la liste de leurs caractéristiques pertinentes, c'est-à-dire permet sa définition adaptée à chaque contexte. Enfin, l'ensemble des outils des sciences sociales, en particulier ceux de l'observation et de l'entretien ethnographiques, doivent permettre une appréhension plus réaliste de l'action publique.

Une dernière remarque s'impose, à propos de la faisabilité de la démarche. La fréquentation simultanée ou à intervalles rapprochés d'agents de l'AAES, de consultants, de médecins, d'infirmières, d'aides-soignantes, de patients, etc., m'a certes permis de mieux cerner les points communs et les différences de leurs regards sur les hôpitaux. Au bout d'un certain temps, j'avais même constamment en tête, quand j'étais sur un type de terrain - agence, sociétés de conseil, services de soin, patients - le regard que les membres des autres pourraient porter dessus. Mais ceci, étant donné leurs conflits, a parfois confiné à la schizophrénie. Si on ajoute à cela le fait qu'il est difficile, au milieu de ces heurts d'intérêts, de rester totalement neutre, et que par conséquent certains enquêtés ne m'ont finalement pas été particulièrement sympathiques (Avanza, 2008), on peut se faire une idée du malaise parfois éprouvé au cours de l'enquête. C'est pourquoi l'ethnographie appliquée à l'étude des politiques publiques donnerait peut-être ses meilleurs résultats si elle était pratiquée en équipe. Certes, chaque enquêteur ne verrait directement qu'une partie de l'objet et courrait ainsi le risque de ne pas suivre sur son terrain les pistes suggérées par ceux des autres. Mais les membres du groupe pourraient se répartir les sites à investiguer, ce qui présenterait plusieurs avantages. D'une part, cela simplifierait considérablement la gestion des rôles multiples, et permettrait notamment d'éviter des télescopages de scènes sociales (par exemple, entre soignants en grève et directeurs d'hôpital tentant de réprimer cette grève - moi-même devant être proche de ces deux 
groupes en même temps). D'autre part, cela déplacerait le conflit de l'intérieur d'un enquêteur vers le groupe dans son ensemble, où il devrait pouvoir s'exprimer par des discussions menées dans un respect mutuel et qui contribueraient probablement à faire avancer la réflexion.

Lors d'une journée d'étude sur l'apport possible de l'ethnographie à sa discipline, un politiste affirmait que l'" on ne peut pas être Lawrence d'Arabie ", pointant tant des difficultés liées à la définition de soi analogues à celles que l'on vient de voir, que l'ampleur de la tâche face au format limité des travaux académiquement exigés ${ }^{23}$. II est tentant à présent de répondre, outre le fait que la définition des normes académiques est le résultat toujours provisoire de rapports de force mouvants, que certains travaux mentionnés ici ont fait la preuve que ces difficultés pouvaient être surmontées, et qu'au demeurant des "ficelles" sont là pour faciliter la tâche. Si Lawrence d'Arabie demeure un idéal méthodologique inaccessible, au moins peut-on tenter de s'en rapprocher.

\author{
Nicolas Belorgey \\ PRISME-GSPE (UMR7012) \\ nicolas.belorgey@misha.fr
}

\title{
Bibliographie
}

AVANZA, M. (2008), "Comment faire de l'ethnographie quand on n'aime pas "ses indigènes" ? Enquête au sein d'un mouvement xénophobe ", dans FASSIN, D., BENSA, A., (dir.), Les Politiques de l'enquête, épreuves ethnographiques, Paris, La Découverte, p. 41-58.

AVANZA, M., LAFERTE, G. (2005), "Dépasser la "construction des identités" ? Identification, image sociale, appartenance ", Genèses, 61 (4), p. 134-152.

AVRIL, C., CARTIER, M., SERRE, D. (2010), Enquêter sur le travail : concepts, méthodes, récits, Paris, La Découverte.

AVRIL, C., CARTIER, M., SIBLOT, Y. (2005), «Les rapports aux services publics des usagers et agents de milieux populaires: quels effets des réformes de modernisation ", Sociétés contemporaines, 58, p. 5-18.

BASZANGER, I., DODIER, N. (1997), "Totalisation et altérité dans l'enquête ethnographique ", Revue française de sociologie, 38 (1), p. 37-66.

BATESON, G. (1971) [1936], La Cérémonie du naven : les problèmes posés par la description sous trois rapports d'une tribu de Nouvelle-Guinée, Paris, Minuit.
BEAUD, S., WEBER, F. (2010) [1997], Guide de l'enquête de terrain : produire et analyser des données ethnographiques, Paris, La Découverte.

BECKER, H. (1985) [1963], Outsiders. Études de sociologie de la déviance, Paris, Métailié.

BECKER, H. (2004), Écrire les sciences sociales, Paris, Economica.

BELORGEY, N. (2009), " "J'oublie de prendre mes médicaments". Les variations de l'observance dans un réseau de soins", Sociologie santé, 30, p. 287-310.

BELORGEY, N. (2010), L'Hôpital sous pression: enquête sur le "nouveau management public", Paris, La Découverte.

BELORGEY, N. (2011a), « Les cadres de l'expertise publique. Les trois dimensions de l'expertise d'État saisies par une ethnographie des économistes de la Banque de France ", Genèses, 85, p. 93-114.

BELORGEY, N. (2011b), "Réduire le temps d'attente et de passage aux urgences". Une entreprise de "réforme" d'un service public et ses effets sociaux", Actes de la recherche en sciences sociales, 189, p. 19-35.

BENAMOUZIG, D. (2005), La Santé au miroir de l'économie, Paris, PUF.

23. "Quel statut pour l'observation ethnographique dans l'écriture en science politique ? ", Journée d'étude du Crapul, Lausanne, 4-5 mai 2009. Ce texte est très redevable aux participants à cette journée, en particulier à ses organisateurs, Martina Avanza et Alexandre Lambelet. 
BERTAUX, D. (1996), Les Récits de vie, Paris, Nathan.

BERTAUX-WIAME, I. (2004), « Devenir indépendant, une affaire de couple", Cahiers du genre, 37, p. 13-40.

BESSIÈRE, C. (2003), "Race/classe/genre. Parcours dans l'historiographie américaine des femmes du Sud autour de la guerre de Sécession. " Clio, 17 (1), p. 13.

BEZES, P. (2009), Réinventer l'État, Paris, PUF.

BEZES, P., PIERRU, F. (2012), «État, administration et politiques publiques: les dé-liaisons dangereuses ", Gouvernement \& action publique, 2, p. 41-87.

BILAND, E. (2008), Concours territoriaux et institutionnalisation de l'emploi public local (années 1970-années 2000), thèse sous la direction de Michel Offerlé, Paris, EHESS.

BLAU, P. (1963) [1955], The Dynamics of Bureaucracy. A Study of Interpersonal Relations in two Government Agencies, Chicago (III.), University of Chicago Press.

BLOCH, M. (1990) [1946], L'Étrange défaite, Paris, Gallimard.

BOLTANSKI, L. (1982), Les Cadres: la formation d'un groupe social, Paris, Minuit.

BONGRAND, P., LABORIER, P. (2005), "L'entretien dans l'analyse des politiques publiques: un impensé méthodologique?", Revue française de science politique, 55, p. 73-111.

BOURDIEU, P. (1990), " Droit et passe-droit ", Actes de la recherche en sciences sociales, 81 (1), p. 86-96.

BOURDIEU, P. (dir.) (1993), La Misère du monde, Paris, Seuil.

BOURDIEU, P. (2003), "L'objectivation participante ", Actes de la recherche en sciences sociales, 150 (1), p. 43-58.

BOURGOIS, P. (2001), En quête de respect: le crack à New York, Paris, Seuil.

BRODKIN, E. (2011), "Policy Work: Street-Level Organizations Under New Managerialism ", Journal of Public Administration Research and Theory, 21 (Supplement 2), p. 253-277.

BURAWOY, M. (1998), "The Extended Case Method ", Sociological theory, 16 (1), p. 4-33.

CAMBROSIO, A., LIMOGES, C., PRONOVOST, D. (1990), "Representing Biotechnology: An Ethnography of Quebec Science Policy ", Social Studies of Science, 20 (2), p. 195-227.

CARTIER, M. (2003), Des facteurs et leurs tournées, Paris, La Découverte.
CASTEL, P. (2005), « Le médecin, son patient et ses pairs. Une nouvelle approche de la relation thérapeutique ", Revue française de sociologie, 3, p. 443-467.

CHAUNCEY, G. (2003) [1994], Gay New York: 1890-1940, Paris, Fayard.

COHEN, M.D., MARCH, J.G., OLSEN, J.P. (1972), "A Garbage Can Model of Organizational Choice ", Administrative Science Quarterly, 17 (1), p. 1-25.

CROZIER, M. (1993) [1963], Le Phénomène bureaucratique: essai sur les tendances bureaucratiques des systèmes d'organisation modernes et sur leurs relations en France avec le systèmes social et culturel, Paris, Seuil.

DEMEULENAERE, P. (1996), Homo oeconomicus. Enquête sur la constitution d'un paradigme, Paris, PUF.

DESCOLA, P. (1994), Les Lances du crépuscule, Paris, Plon.

DODIER, N., CAMUS, A. (1997), «L'admission des malades. Histoire et pragmatique de l'accueil à l'hôpital ", Annales HSS, 4, p. 733-763.

DUBOIS, V. (2003) [1997], La Vie au guichet. Relation administrative et traitement de la misère, Paris, Economica.

DUBOIS, V. (2009), "L'action publique", dans COHEN, A., LACROIX, B., RIUTORT, P. (dir.), Nouveau manuel de science politique, Paris, La Découverte, p. 311-330.

DUBOIS, V. (2012), Ethnographier l'action publique : les transformations de l'État social au prisme de l'enquête de terrain ", Gouvernance \& action publique, 1, p. 83-102.

DUBOIS, V., DULONG, D. (2003), " Les ruses de la raison juridique", Recherches et prévisions, 73, p. 53-64.

DURKHEIM, É. (1993) [1895], Les Règles de la méthode sociologique, Paris, PUF.

DUVAL, J. (2002), "Une réforme symbolique de la Sécurité sociale. Les médias et le "trou de la Sécu" ", Actes de la recherche en sciences sociales, 143, p. 53-67.

DUVAL, J. (2007), Le Mythe du "trou de la Sécu ", Paris, Raisons d'agir.

ELIAS, N. (1991), Qu'est-ce que la sociologie?, Paris, Pocket.

ELIAS, N., SCOTSON, J. (1997) [1965], Logiques de l'exclusion. Enquête sociologique au cœur des problèmes d'une communauté, Paris, Fayard.

EVANS-PRITCHARD, E. (1994) [1937], Les Nuer: description des modes de vie et des institutions politiques d'un peuple nilote, Paris, Gallimard.

FAMA, E. (1980), "Agency Problems and the Theory of the Firm ", The Journal of Political Economy, 88 (2), p. 288-307. 
FASSIN, D., FASSIN, É. (dir.) (2006), De la question sociale à la question raciale ? Représenter la société française, Paris, La Découverte.

FOURCAUT, A. (dir.) (1992), Banlieue rouge, 1920-1960, Paris, Autrement.

FOURNIER, P. (2006), "Le sexe et l'âge de l'ethnographe: éclairants pour l'enquêté, contraignants pour l'enquêteur » [http://ethnographiques.org], 11.

FREIDSON, E. (1984) [1970], La Profession médicale, Paris, Payot.

GODECHOT, O. (2006), "Hold-up en finance", Revue française de sociologie, 47 (2), p. 341-371.

GODECHOT, O. (2007), Working rich: Salaires, bonus et appropriation du profit dans l'industrie financière, Paris, La Découverte.

GRIGNON, C., PASSERON, J.-C. (1989), Le Savant et le Populaire : misérabilisme et populisme en sociologie et en littérature, Paris, Gallimard-Seuil.

GUSFIELD, J.R. (1986) [1966], Symbolic Crusade: Status Politics and the American Temperance movement, Urbana (III.), University of Illinois Press.

HASSENTEUFEL, P. (2008), Sociologie politique: l'action publique, Paris, Armand Colin.

HASSENTEUFEL, P., SMITH, A. (2002), "Essoufflement ou second souffle? L'analyse des politiques publiques "à la française" ", Revue française de science politique, 52 (1), p. 53-73.

HMED, C. (2007), « Contester une institution dans le cas d'une mobilisation improbable: la "grève des loyers" dans les foyers Sonacotra dans les années 1970 ", Sociétés contemporaines, 65 (1), p. 55-81.

HUGHES, E. (1984) [1946], "The Knitting of Racial Groups in Industry ", in The Sociological Eye, New Brunswick \& London, Transaction Books, p. 265-275.

HUGHES, E. (1984) [1946], "Social Role and the Division of Labour ", in The Sociological Eye, New Brunswick \& London, Transaction Books, p. 304-310.

JAMOUS, H. (1969), Sociologie de la décision. La réforme des études médicales et des structures hospitalières, Paris, CNRS.

JONES, C. (2002) [1970], An Introduction to the Study of Public Policy, Belmont (Calif.), Wadsworth Pub.

KANTOROWICZ, E. (1989) [1957], Les Deux Corps du roi : essai sur la théologie politique au Moyen Âge, Paris, Gallimard.

KERGOAT, D., IMBERT, F., LE DOARE, H., SENOTIER, D. (1992), Les Infirmières et leur coordination, 1988-1989, Paris, Lamarre.

KOLM, S.-C. (1986), Philosophie de l'économie, Paris, Seuil.
LABOV, W. (1978) [1972], Le Parler ordinaire: la langue dans les ghettos noirs des États-Unis, Paris, Minuit.

LASCOUMES, P. (1990), "Normes juridiques et mise en œuvre des politiques publiques ", L'Année sociologique, 40, p. 43-71.

LASCOUMES, P., LE GALES, P. (2007), Sociologie de l'action publique, Paris, Armand Colin.

LASSWELL, H. (1956), The Decision Process: Seven Categories of Functional Analysis, Bureau of Governmental Research, College of Business and Public Administration, University of Maryland.

LATOUR, B. (1988), " Le Grand Partage ", La Revue du Mauss, 1, p. 27-64

LATOUR, B. (2002), La Fabrique du droit : une ethnographie du Conseil d'État, Paris, La Découverte.

LAURENS, S. (2007), "Pourquoi et comment poser les questions qui fâchent. Réflexions sur les dilemmes récurrents que posent les entretiens avec les imposants ", Genèses, 69 (4), p. 112-127.

LAURENS, S. (2009), Une politisation feutrée, Paris, Belin.

LENCLUD, G. (1992), "Le Grand partage ou la tentation ethnologique", Cahiers d'ethnologie de la France, 7, p. 9-39.

LENOIR, R. (1993), "Un reproche vivant ", dans BOURDIEU, P. (dir.), La Misère du monde, Paris, Seuil, p. 299-315.

LEVI-STRAUSS, C. (1989) [1952], Race et Histoire, Paris, Unesco-Gallimard.

LEVI-STRAUSS, C. (1990), La Pensée sauvage, Paris, Plon.

LIGNIER, W. (2008), " La barrière de l'âge. Conditions de l'observation participante avec des enfants ", Genèses, 73, p. 20-36.

LINDBLOM, C. (1959), "The Science of Muddling Through", Public Administration Review, 19 (3), p. $79-88$.

LINHART, R. (1981) [1978], L'Établi, Paris, Minuit.

LIPSKY, M. (1980), Street-Level Bureaucracy. Dilemma of the Individual in the Public Service, New York (N. Y.), Russel Sage Foundation.

MAGET, M. (1953), Guide d'étude directe des comportements culturels, Paris, CNRS.

MALINOWSKI, B. (1989) [1922], Les Argonautes du Pacifique occidental, Paris, Gallimard.

MARCH, J., SIMON, H. (1958), Organizations, New York (N. Y.), John Wiley \& Sons.

MARCUS, G. (1998), «Ethnography in/of the World System: The Emergence of Multi-sited Ethnography ", in Ethnography through thick and thin, 
Princeton (N. J.), Princeton University Press, p. 79-104.

MAUGER, G. (1991), "Enquêter en milieu populaire ", Genèses, 6 (1), p. 125-143.

MAUSS, M. (1980) [1923], "Essai sur le don. Formes et raison de l'échange dans les sociétés archaïques ", dans Sociologie et Anthropologie, Paris, PUF, p. 145-284.

MULLER, P. (2009) [1990], Les Politiques publiques, Paris, PUF.

MUSSELIN, C. (2005), "Sociologie de l'action organisée et analyse des politiques publiques: deux approches pour un même objet ? ", Revue française de science politique, 55 (1), p. 51-71.

NAY, O., SMITH, A. (dir.) (2002), Le Gouvernement du compromis, Paris, Economica.

OFFERLÉ, M., LAGROYE, J. (dir.) (2010), Sociologie de l'institution, Paris, Belin.

OLIVIER DE SARDAN, J.-P. (1995), "La politique du terrain, sur la production des données en anthropologie ", Enquête, 1, p. 71-100.

OLIVIER DE SARDAN, J.-P. (2000), « Le "je" méthodologique. Implication et explicitation dans l'enquête de terrain ", Revue française de sociologie, 41 (3), p. 417-445.

PAILLET, A. (2007), Sauver la vie, donner la mort. Une sociologie de l'éthique en réanimation médicale, Paris, La Dispute.

PARSONS, W. (1995), Public Policy: An Introduction to the Theory and Practice of Policy Analysis, Aldershot, Edward Elgar Dorey.

PASSERON, J.-C. (2006) [1991], Le Raisonnement sociologique, Paris, Nathan.

PIERRU, F. (2007), Hippocrate malade de ses réformes, Paris, Éditions du croquant.

PRESSMAN, J., WILDAVSKY, A. (1973), Implementation. How Great Expectations in Washington are Dashed in Oakland, Berkeley \& Los Angeles (Calif.), University of California Press.

REVEL, J. (1996), Jeux d'échelles : la micro-analyse à l'expérience, Paris, Seuil.

SABATIER, P. (1986), "Top-down and Bottom up Approaches to Implementation Research: A Critical Analysis and Suggested Synthesis ", Journal of Public Policy, 6, p. 21-48.

SAHLINS, M. (1990) [1972], Âge de pierre, âge d'abondance: l'économie des sociétés primitives, Paris, Gallimard.

SCHWARTZ, O. (1993), "Postface: L'empirisme irréductible ", dans ANDERSON, N., Le Hobo, sociologie du sans-abri, Paris, Nathan, p. 265-308.
SERRE, D. (2001), "La "judiciarisation" en actes", Actes de la recherche en sciences sociales, 136/137, p. 70-82.

SERRE, D. (2009), Les Coulisses de l'État social: enquête sur les signalements d'enfant en danger, Paris, Seuil.

SFEZ, L. (1992) [1973], Critique de la décision, Paris, Presses de la FNSP

SIBLOT, Y. (2006), Faire valoir ses droits au quotidien. Les services publics dans les quartiers populaires, Paris, Presses de Sciences po.

SIMEANT, J. (1998), La Cause des sans-papiers, Paris, Presses de Sciences po.

SORIGNET, P.-E. (2010), Danser : enquête dans les coulisses d'une vocation, Paris, La Découverte.

SOULE, B. (2007), "Observation participante ou participation observante? Usages et justifications de la notion de participation observante en sciences sociales ", Recherches qualitatives, 27 (1), p. 127-140.

SPIRE, A. (2005), Étrangers à la carte : l'administration de l'immigration en France, Paris, Grasset.

STACK, C. (1997), « What are Given as Givens: Ethnography and Critical Policy Studies », Ethos, 25 (2), p. 191-207.

TOPALOV, C. (1999), Laboratoires du nouveau siècle: la nébuleuse réformatrice et ses réseaux en France, 1880-1914, Paris, EHESS.

WACQUANT, L. (2002) [2000], Corps et Âme, Paris, Agone.

WARIN, P. (1999), "Les "ressortissants" dans les analyses des politiques publiques ", Revue française de science politique, 1, p. 103-121.

WARIN, P. (2002), Les Dépanneurs de justice. Les petits fonctionnaires entre qualité et équité, Paris, LGDJ.

WEBER, F. (1989), Le Travail à-côté : étude d'ethnographie ouvrière, Paris, INRA-EHESS.

WEBER, F. (2003), " Postface à la deuxième édition. Actualité de la recherche ethnographique ", dans BEAUD, S., WEBER, F., Manuel de l'enquête de terrain, Paris, La Découverte, p. 315-340.

WEBER, F. (2005), Le Sang, le nom, le quotidien. Une sociologie de la parenté pratique, Paris, Aux lieux d'être.

WEBER, F. (2009), Manuel de l'ethnographe, Paris, PUF.

WEBER, M. (1995) [1922], Économie et Société, Paris, Presses Pocket.

WEINER, A. (1983) [1976], La Richesse des femmes ou Comment l'esprit vient aux hommes: îles Trobriand, Paris, Seuil.

WELLER, J.-M. (1999), L'État au guichet, Paris, Desclée de Brouwer. 